\title{
Submergence of the filamentous Zygnematophyceae Mougeotia induces differential gene expression patterns associated with core metabolism and photosynthesis
}

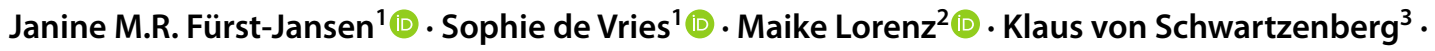 \\ John M. Archibald ${ }^{4}$. Jan de Vries ${ }^{1,5,6}$
}

Received: 16 June 2021 / Accepted: 6 December 2021/ Published online: 22 December 2021

(c) The Author(s) 2021

\begin{abstract}
The streptophyte algal class Zygnematophyceae is the closest algal sister lineage to land plants. In nature, Zygnematophyceae can grow in both terrestrial and freshwater habitats and how they do this is an important unanswered question. Here, we studied what happens to the zygnematophyceaen alga Mougeotia sp., which usually occurs in permanent and temporary freshwater bodies, when it is shifted to liquid growth conditions after growth on a solid substrate. Using global differential gene expression profiling, we identified changes in the core metabolism of the organism interlinked with photosynthesis; the latter went hand in hand with measurable impact on the photophysiology as assessed via pulse amplitude modulation (PAM) fluorometry. Our data reveal a pronounced change in the overall physiology of the alga after submergence and pinpoint candidate genes that play a role. These results provide insight into the importance of photophysiological readjustment when filamentous Zygnematophyceae transition between terrestrial and aquatic habitats.
\end{abstract}

Keywords Streptophyte algae $\cdot$ Charophytes $\cdot$ RNAseq $\cdot$ Algal culturing $\cdot$ Algal physiology

Handling Editor: Andreas Holzinger

Jan de Vries

devries.jan@uni-goettingen.de

Janine M.R. Fürst-Jansen

Janine.fuerst-jansen@uni-goettingen.de

Sophie de Vries

sophie.devries@uni-goettingen.de

Maike Lorenz

mlorenz@uni-goettingen.de

Klaus von Schwartzenberg

Klaus.von.Schwartzenberg@uni-hamburg.de

John M. Archibald

john.archibald@dal.ca

1 Department of Applied Bioinformatics, Institute for Microbiology and Genetics, University of Goettingen, Goldschmidtstr. 1, University of Goettingen, 37077 Goettingen, Germany

2 Department of Experimental Phycology and SAG Culture Collection of Algae, Albrecht-von-Haller Institute for Plant
Science, University of Goettingen, Nikolausberger Weg 18, 37073 Goettingen, Germany

3 Institute of Plant Science and Microbiology, Microalgae and Zygnematophyceae Collection Hamburg (MZCH) and Aquatic Ecophysiology and Phycology, Universität Hamburg, Ohnhorststr. 18, 22609 Hamburg, Germany

4 Department of Biochemistry and Molecular Biology, Dalhousie University, Sir Charles Tupper Medical Building, 5850 College Street, Halifax, NS B3H 4R2, Canada

5 Goettingen Center for Molecular Biosciences (GZMB), University of Goettingen, Justus-von-Liebig-Weg 11, 37077 Goettingen, Germany

6 Campus Institute Data Science (CIDAS), University of Goettingen, Goldschmidstr. 1, 37077 Goettingen, Germany 


\section{Introduction}

Streptophyte algae diverged from the chlorophytes and prasinodermophytes between 700 and 1000 million years ago (Zimmer et al. 2007; Morris et al. 2018; Li et al. 2020). They form a paraphylum that is sister to the monophyletic Embryophyta, the land plants-together, land plants and streptophyte algae form the monophylum Streptophyta (Wickett et al. 2014). One of the most important questions in the field of land plant evolution is which particular lineage of streptophyte algae within this paraphylum represents the sister lineage to land plants. Streptophyte algae encompass a diverse range of organisms, including the unicellular Mesostigmatophyceae and Chlorokybophyceae (cell packages), consisting of only a few species (see also Irisarri et al. 2021), the unicellular and filamentous Klebsormidiophyceae (Mikhailyuk et al. 2015), and the streptophyte algae within Phragmoplastophyta that include morphologically complex multicellular organisms such as the Charophyceae-and the land plants. Various lines of evidence indicate that, among these Phragmoplastophyta, the Zygnematophyceae represent the sister lineage to land plants (Wodniok et al. 2011; Wickett et al. 2014; LeebensMack et al. 2019). It is thus of considerable interest what physiological properties these organisms possess-combined with data on land plants, such an understanding makes it possible to infer the physiology of the earliest land plants (Fürst-Jansen et al. 2020).

A key piece of the puzzle of understanding plant terrestrialization is the difference between growth in an aquatic environment and growth in a terrestrial habitat with limited water supply. Throughout the course of evolution, various algal lineages have mastered this so-called wet-to-dry transition. This is no small feat. The terrestrial habitat poses various challenges for a photosynthesizing organism, including fluctuations in abiotic factors such as temperature, water availability, and intensity and quality of irradiance (Foyer et al. 1994; Karsten et al. 2007; Holzinger et al. 2014; Ohama et al. 2017).

Terrestrial algae meet the challenges of their habitat with various physiological adaptations (Holzinger and Pichrtová 2016). These include the presence of mycosporine-like amino acids (MAAs) found in both chlorophyte and streptophyte algae. MAAs have UV-protecting properties. Among streptophyte algae, the Klebsormidiophyceae Hormidiella and Klebsormidium stand out by producing potent sunscreen MAAs with an absorption maximum at 325 and $324 \mathrm{~nm}$ (Kitzing and Karsten 2015). While such MAAs have not been reported for Zygnematophyceae, Zygnema spp. are known to produce phenolic compounds upon elevated UV irradiance (Pichrtová et al. 2013). Indeed, the unicellular Zygnematophyceae Penium margaritaceum was recently reported to contain flavonoids (Jiao et al. 2020). While the exact biochemical routes towards these metabolites are currently elusive, homologs of genes coding for core enzymatic biosynthetic steps that lead to relevant precursor metabolites in land plants (the phenylpropanoid pathway sensu lato) are also found in streptophyte algae (de Vries et al. 2017, 2021). Recently, Renault et al. (2019) highlighted the putative links between phenylpropanoid biosynthesis in streptophyte algae and shared ancestral chassis for producing hydrophobic polymers from which lignin, cutin, suberin, and sporopollenin arose. Indeed, Zygnematophyceae surround their zygotes with resistant polymers resembling sporopollenin (de Vries et al. 1983; Poulícková et al. 2007). Recently, Permann et al. (2021) employed glycan labeling as well as Raman spectroscopy to zygospores of Mougeotia disjuncta (which belongs to the same algal genus as the strains analyzed here); they found these zygospores to consist of a combination of carbohydrates, lipids, and aromatic compounds, speaking to sporopollenin-like material.

UV irradiance is not the only sunlight-associated challenge in the terrestrial habitat. Photosynthetically active radiance (PAR) reaches much higher levels on the surface of the earth as opposed to an aquatic environment, where the sunlight is buffered by the absorptive properties of water. One of the main mechanisms that mitigates damage to the components of the photosynthetic light reaction, in particular the vulnerable photosystem II, is non-photochemical quenching (NPQ; Müller et al. 2001; Jahns and Holzwarth 2012). The first and fastest response of NPQ is energy-dependent quenching (qE). Its activation hinges upon conformational changes in the photosystem and the detection of an altered $\mathrm{pH}$ in the thylakoid lumen (Krause et al. 1982). While their predominance varies across the green lineage, evidence suggests that the LHCSR (light-harvesting complex stress-related protein) and/or PSBS (photosystem II subunit $\mathrm{S}$ ) proteins play a major role in this process $(\mathrm{Li}$ et al. 2000; Peers et al. 2009; Gerotto and Morosinotto 2013; Correa-Galvis et al. 2016). It is nevertheless prudent to note that some chlorophyte algae seem to lack qE (Christa et al. 2017). The result of NPQ is that superfluous energy, which cannot be meaningfully channeled into the light reaction chain, dissipates as harmless heat.

The role of NPQ and acclimation processes of the photosystem has been extensively studied in terrestrial streptophyte algae. For example, Herburger and Holzinger (2015) found that the photosynthetic effective quantum yield is strongly reduced in Klebsormidium strains upon desiccation but also recovers fully upon rehydration suggesting a high desiccation tolerance. Furthermore, Karsten et al. (2014) found that the sister group of Klebsormidium, Interfilum, also appears to have similar characteristics regarding high tolerance to stressors such as desiccation but also 
temperature that reflect in their photosynthetic physiology. That said, not only the family of Klebsormidiaceae shows this high tolerance to stressors. In the class of Zygnematophyceae, Holzinger et al. (2018) found that after UV-treatment in different Zygnema strains their effective quantum yield recovers completely in some cases. There are however other conserved mechanisms for photoprotection acting in algae. One is the expression of EARLY LIGHT INDUCED PROTEIN (ELIP). ELIPs are chlorophyll $a / b$-binding proteins that accumulate under stress and have a photoprotective function (Montané et al. 1997; Hutin et al. 2003). Elevated expression of ELIP-coding genes under light and temperature stress has now been reported for the Zygnematophyceae Zygnema and Mougeotia (de Vries et al. 2018; Rippin et al. 2019; de Vries et al. 2020). As with the relevance of NPQ under water scarcity, ELIP expression is also induced in desiccated Zygnema (Rippin et al. 2017). Thus, while we know about physiological responses of Zygnematophyceae challenged with water scarcity, we know very little about the reverse process-which is of similar importance for organisms that thrive in temporary water bodies. Plant terrestrialization likely entailed a repetition of several wet-todry and dry-to-wet transitions; therefore, investigating both transitions is important. Furthermore, living on land means a steady change between wet and dry conditions (rain, fog, and dew). Mougeotia spp. live in a variety of freshwater habitats, many of them are temporary habitats such as ditches and small temporary ponds.

In this study, we have used a laboratory controlled environmental shift approach to emulate what happens to the filamentous zygnematophyceaen alga Mougeotia sp., which predominantly lives in freshwater habitats, shortly after being submerged. Our data highlight photosynthesis-associated physiological responses and the global gene expression patterns that bring them to bear.

\section{Material and methods}

\section{Culturing and treatment}

For the RNAseq experiments, Mougeotia sp. MZCH 240 (which we obtained from the Microalgae and Zygnematophyceae Collection, Hamburg, Germany, [von Schwartzenberg et al. 2013]) was cultured as described in de Vries et al. (2020) - algae were grown for 7 days on modified freshwater $\mathrm{F} / 2$ (Guillard 1975) with $1 \%$ agar at $22^{\circ} \mathrm{C}$ and $120 \mu \mathrm{mol}$ quanta $\mathrm{m}^{-2} \mathrm{~s}^{-1}$ from an LED light source $(12 \mathrm{~h} / 12 \mathrm{~h}$ light/dark cycle) in $9 \mathrm{~cm}$ plates. For submergence, $10 \mathrm{~mL}$ of temperature-adjusted liquid freshwater F/2 (Guillard 1975) were added to each agar plate; for RNAseq, algae were directly transferred into Trizol (Thermo Fisher, Walthm, MA, USA) after $4 \mathrm{~h}$ of submergence.
For the photophysiological experiments, Mougeotia scalaris strain SAG 164.80 (of the Culture Collection of Algae, Göttingen, Germany; Friedl and Lorenz 2012) and Mougeotia sp. MZCH 240 were grown on (i) WHM medium (M. scalaris SAG 164.80; Nichols 1973) with 1\% agar or in liquid WHM medium or (ii) modified freshwater F/2 (Mougeotia sp. MZCH 240) with 1\% agar or in liquid F/2 medium at $22^{\circ} \mathrm{C}$ and $120 \mu \mathrm{mol}$ quanta $\mathrm{m}^{-2} \mathrm{~s}^{-1}$ from an LED light source $(12 \mathrm{~h} / 12 \mathrm{~h}$ light/dark cycle). For submergence, $10 \mathrm{~mL}$ of temperature-adjusted liquid WHM (M. scalaris SAG 164.80) or liquid F/2 (Mougeotia sp. MZCH 240) were added to each plate and $F_{\mathrm{v}} / F_{\mathrm{m}}$ was measured after various incubation timepoints $(2 \mathrm{~h}, 4 \mathrm{~h}, 6 \mathrm{~h}, 8 \mathrm{~h}, 24 \mathrm{~h}$; plus $1 \mathrm{~h}$ and $3 \mathrm{~h}$ for Mougeotia scalaris SAG 164.80). For morphological observations and micrographs, M. scalaris SAG 164.80 and Mougeotia sp. SAG 650-1 were used as additional comparative material and grown either in liquid or solid for 9 weeks on Desmidiacean Medium (MiEB12; medium 7 of Schlösser 1994). For Mougeotia sp. MZCH 240, microscope pictures were taken after the $24 \mathrm{~h}$ timepoint under the growing conditions described above.

\section{RNA extraction and sequencing}

RNA extraction and sequencing procedures were described in de Vries et al. (2020). In brief, we extracted RNA in six biological replicates from the control samples and in biological triplicates from the liquid-treated samples. For RNA extraction, algae were directly transferred into $1 \mathrm{~mL}$ of Trizol using a sterilized spatula (Thermo Fisher, Waltham, MA, USA); extraction procedures were carried out in accordance to the protocol provided by the manufacturer. Isolated RNA was treated with DNAse I (Thermo Fisher), quality assessed on a formamide agarose gel, quantified using a Nanodrop spectrometer (Thermo Fisher), and shipped to Genome Québec (Montreal, Canada) for sequencing. There, RNA was quality checked again, using a Bioanalyzer (Agilent Technologies Inc., Santa Clara, CA, USA). Libraries were constructed using the NEB mRNA stranded Library preparation kit (New England Biolabs, Beverly, MA, USA), on the Illumina NovaSeq6000 platform.

\section{RNAseq analyses: data processing, statistics, KEGG, and GOterm}

Initial processing of the RNAseq data was described in de Vries et al. (2020). In brief, reads were checked for quality using FASTQC version 0.11.7 (FASTQC 2018), trimmed with TRIMMOMATIC v0.36 (Bolger et al. 2014; settings: ILLUMINACLIP:TruSeq3-PE- 2.fa:2:30:10:2:TRUE HEADCROP:10 TRAILING:3 SLIDINGWINDOW:4:20 MINLEN:36), and quality checked again using FASTQC v0.11.7. For details on read data, see the "Data availability" 
section. The transcriptome assembly using the TRINITY pipeline (Haas et al. 2013), RSEM (RNA-Seq by Expectation Maximization)-based read mapping (Li and Dewey 2011) was carried out and described in de Vries et al. (2020).

Negative binomial distribution-based statistical analyses of the read counts were performed using edgeR version 3.28.0 (Robinson et al. 2010), taking the biological triplicates into account. For all downstream analyses, only gene expression changes with a Benjamini-Hochberg-corrected $p$ value $\leq 0.001$ and significantly elevated differential gene expression $\left(\log _{2}\right.$ (fold change) $\leq-1$ or $\log _{2}$ (fold change) $\geq$ 1) were considered.

For gene expression analysis based on KEGG orthologs, we worked with expression levels in TPM that were normalized via TMM (trimmed mean of $M$ values; Robinson and Oshlack 2010). These data against KEGG pathways occur in land plants. If multiple transcripts had the same KEGG ortholog as their best hit, their expression values were combined-for the final calculations, a given KEGG ortholog had one TMM-normalized TPM value.

For GO term enrichment using GOrilla (Eden et al. 2009), we used AGI numbers obtained by querying the predicted Mougeotia proteins against Arabidopsis in a BLASTp in a comparison of two unranked list of genes. For this, we used all obtained Arabidopsis homologs (i.e. the best BLASTp hits) as the background set (as the whole transcriptome) and all significantly regulated genes as target set—one target set for all up-regulated genes, one for all downregulated genes.

\section{Photophysiology}

All measurements of the maximum-quantum yield $\left(F_{\mathrm{v}} / F_{\mathrm{m}}\right)$ were done using the maxi version of the Imaging-PAM (ImagMAX/L, M-series, Walz) with an IMAG-K5 CCD camera controlled with the Imaging WinGigE (V2.32) software. Treated as well as control samples were dark adapted 10-30 min before measurement. For $F_{\mathrm{v}} / F_{\mathrm{m}}$ measurement, a short saturation pulse with intensity 10 (setup 1-3; level 3 for setup 4) was applied, which is the standard intensity for the IMAGING-PAM. Within the four experimental setups (three with SAG 164.80 and a fourth with MZCH 240), the settings for measuring light and gain were adjusted slightly (setup 1: measuring light 4, gain 2; setup 2: measuring light 1, gain 10; setup 3(+setup 4): measuring light 1, gain 3 ). A special SP-Routine was not applied to modify the signal to noise ratio of the fluorescence measurement. Statistical analysis was done using Mann-Whitney $U$ tests (Mann and Whitney 1947) with R (version 3.6.1).

\section{Phylogenetic analysis}

To explore whether the ABA3 and PAP homolog we detected in the RNA-Seq-based de novo assembly represents an ABA3 ortholog in Mougeotia sp. MZCH 240, we used BLASTp to mine the protein datasets of (i) the land plants Anthoceros agrestis (Li et al. 2020), Arabidopsis thaliana (Lamesch et al. 2012), Azolla filiculoides (Li et al. 2018), Marchantia polymorpha (Bowman et al. 2017), Nicotiana tabacum (Sierro et al. 2014), Physcomitrium patens (Lang et al. 2018), Selaginella moellendorffi (Banks et al. 2011); (ii) the streptophyte algae Chlorokybus atmophyticus CCAC 0220 (Wang et al. 2020), Chara braunii S 276 and S 277 (Nishiyama et al. 2018), Klebsormidium nitens NIES-2285 (Hori et al. 2014), Mesotaenium endlicherianum SAG 12.97 (Cheng et al. 2019), Mesostigma viride CCAC 1140 (Wang et al. 2020), Spirogloea muscicola CCAC 0214 (Cheng et al. 2019); (iii) Bathycoccus prasinos RCC 1105 (Moreau et al. 2012), Chlamydomonas reinhardtii CC-503 (Merchant et al. 2007), Volvox carteri f. nagariensis, Eve (Prochnik et al. 2010).

All obtained sequences were aligned using MAFFT (Katoh and Standley 2013) with the L-INS-I settings. The alignment was used for computing a maximum likelihood phylogeny using IQ-TREE multicore v.1.5.5 for Linux 64-bit built (Nguyen et al. 2015) with 100 bootstrap replicates; the best model for protein evolution $(\mathrm{WAG}+\mathrm{F}+\mathrm{I}+\mathrm{G} 4$ for $\mathrm{ABA} 3$ and $\mathrm{WAG}+\mathrm{I}+\mathrm{G} 4$ for PAP; both were chosen according to Bayesian Information Criterion) was determined using ModelFinder (Kalyaanamoorthy et al. 2017).

\section{Results and discussion}

\section{Submergence in liquid medium triggers the differential expression of core metabolism and photosynthesis-related genes in Mougeotia sp.}

Using the filamentous zygnematophycean alga Mougeotia sp. (a representative species of the zygnematophycean clade), we analyzed differences in the transcriptome of Mougeotia sp. MZCH 240 under two growth conditions: (i) growth on solid medium and (ii) $4 \mathrm{~h}$ after submergence with liquid medium.

Using the Illumina NovaSeq 6000 platform (operated by Genome Quebec), we obtained $\sim 159$ million paired reads for the solid growth condition (6 biological replicates) and 100 million paired reads for the sample taken $4 \mathrm{~h}$ after submergence (3 biological replicates). After quality checking and trimming, we mapped these reads onto the transcriptome assembly of Mougeotia sp. MZCH 240 (de Vries et al. 2020) using the RSEM toolkit included in the TRINITY pipeline. Using this transcriptome assembly, we worked with 4961 genes, of which 438 genes showed more than 2-fold upregulation and 775 genes showed more than 2-fold downregulation (Figure 1A; more on statistic scrutinization below). 


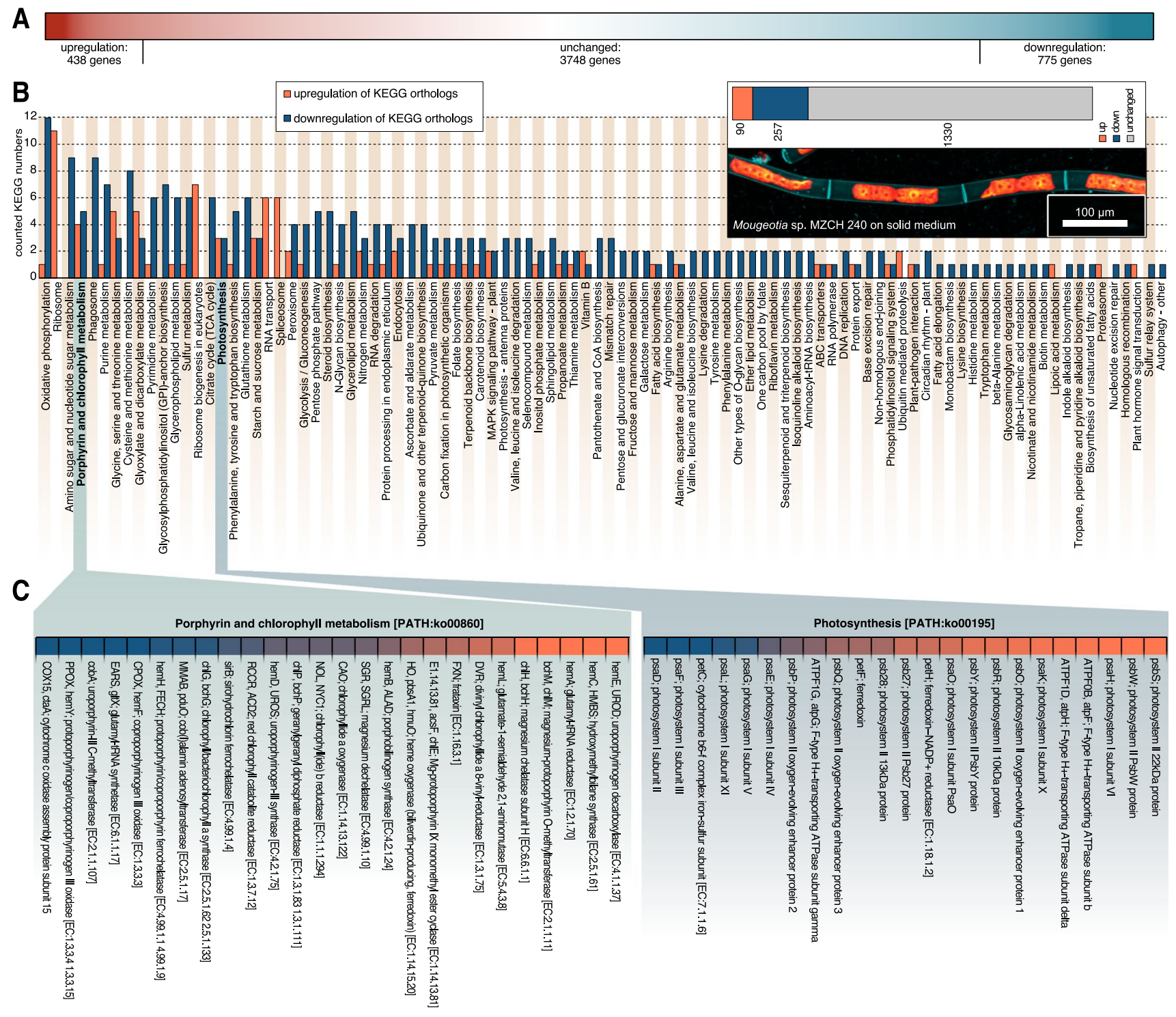

Fig. 1 Global gene expression patterns in Mougeotia sp. MZCH 240. A Gradient-colored depiction (red up-regulated, white unchanged, and blue downregulated genes) of the differential global gene expression profile of all 4961 genes analysed in this study; the differential responses were obtained by comparing global gene expression of Mougeotia sp. MZCH 240 cultured on solid medium and submerged for $4 \mathrm{~h}$ versus control (growth on solid medium). B Gene expression pattern of various KEGG orthologs in Mougeotia sp. MZCH 240. Biological replicates (at least triplicates) of gene expression data $\left(\mathrm{TPM}_{\mathrm{TMM} \text {-normalized }}\right)$ were summed up and set relative to the control condition data (submergence/control) and then mapped against the Kyoto Encyclopedia of Genes and Genomes (KEGG). An up- or downregulation of a KEGG ortholog was considered if it had $a \geq 2$-fold change in gene expression levels. A bar diagram depicts the numbers of all up- (orange) or downregulated (dark blue) KEGG orthologs in the 118 detected KEGG plant pathways in Mougeotia sp. MZCH $2404 \mathrm{~h}$ after being submerged (shift) in liquid medium

First, we were interested in getting an overview over transcriptomic differences induced by submergence in liquid compared to the control culture, which was kept on solid medium. On the upper right side all counted KEGG numbers from up- (90) or down- (257) regulated KEGG orthologs are shown in a stacked bar plot together with 1330 KEGG orthologs with unchanged (grey) gene expression patterns; below is a confocal micrograph of Mougeotia sp. MZCH 240 under control conditions (grown in modified freshwater $\mathrm{F} / 2$ with $1 \%$ agar $22^{\circ} \mathrm{C}$ and $120 \mu$ mol quanta m-2 s-1) - cell walls were made visible using $1 \%$ calcofluor white staining (teal false colored), the plastids are shown in a false-colored red-orange gradient based on their chlorophyll $a$ autofluorescence. C A heatmap of the gene expression patterns in Mougeotia sp. MZCH 240 of the two KEGG plant pathways "Porphyrin and chlorophyll metabolism [PATH:ko00860]" and "Photosynthesis [PATH:ko00195]" in detail. Data is shown as $\log _{2}$ (fold change submergence/control $_{\text {) in a color gradient }}$ ranging from dark blue (downregulation) to orange (upregulation). Unchanged expression levels are not depicted here

medium; we used the pathway framework of the Kyoto Encyclopedia of Genes and Genomes (KEGG) database. 
A

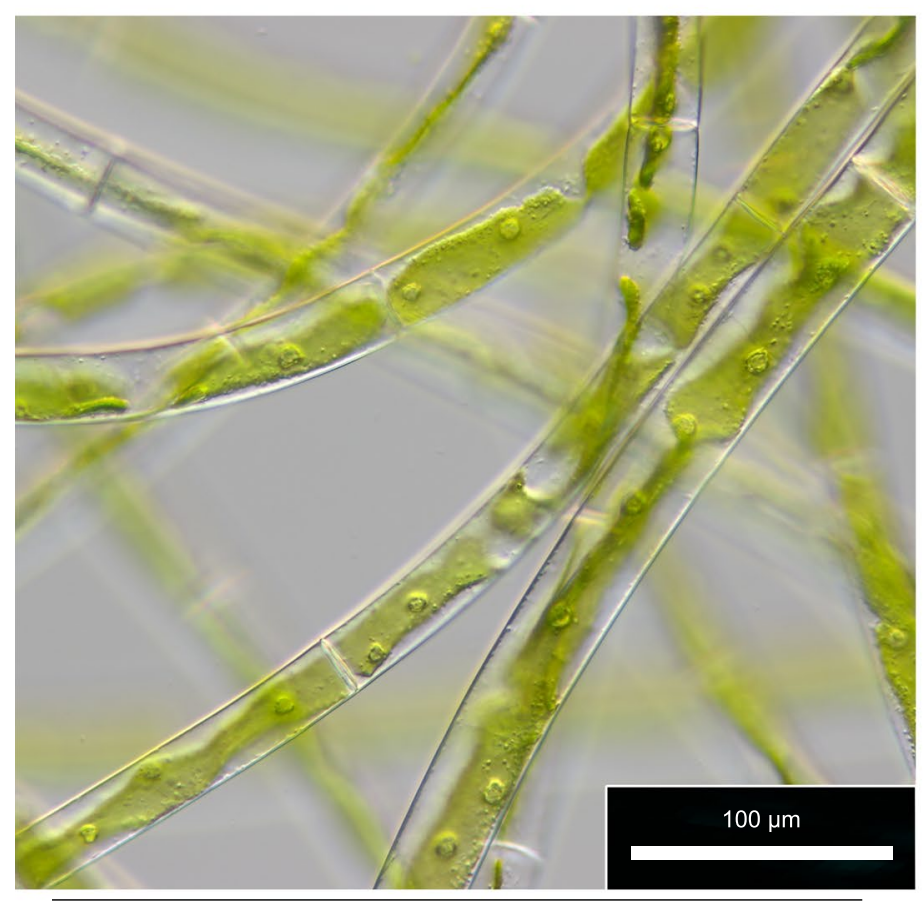

Mougeotia scalaris SAG164.80

C

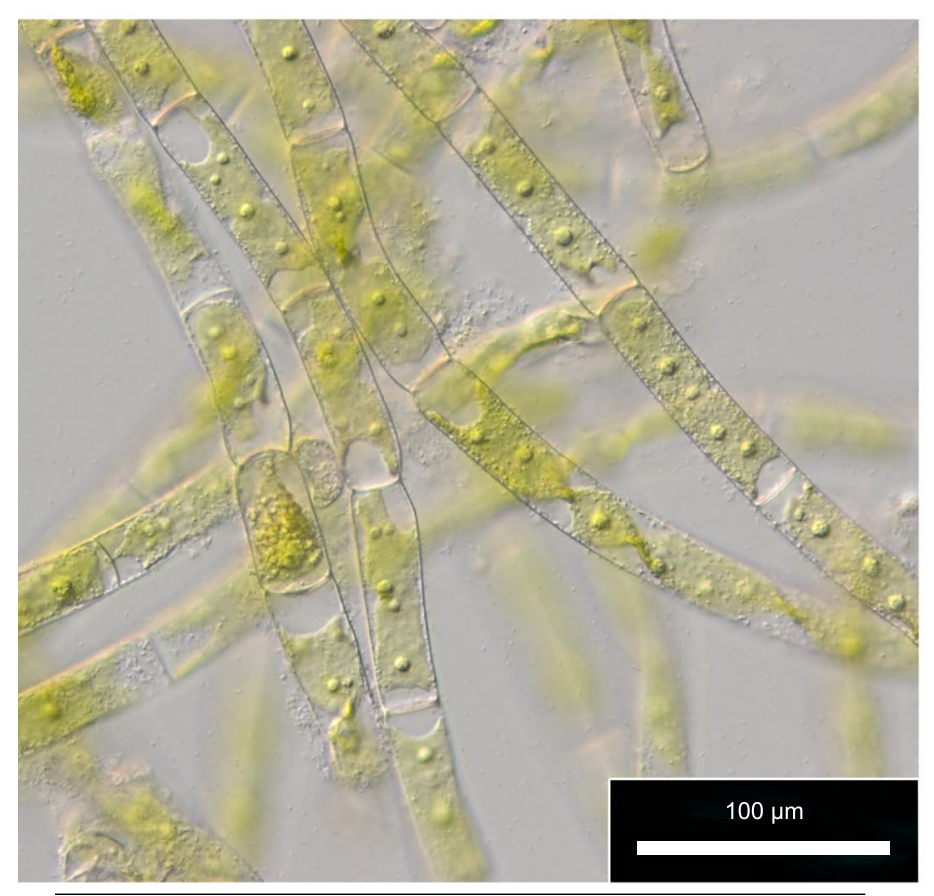

Mougeotia sp. MZCH240

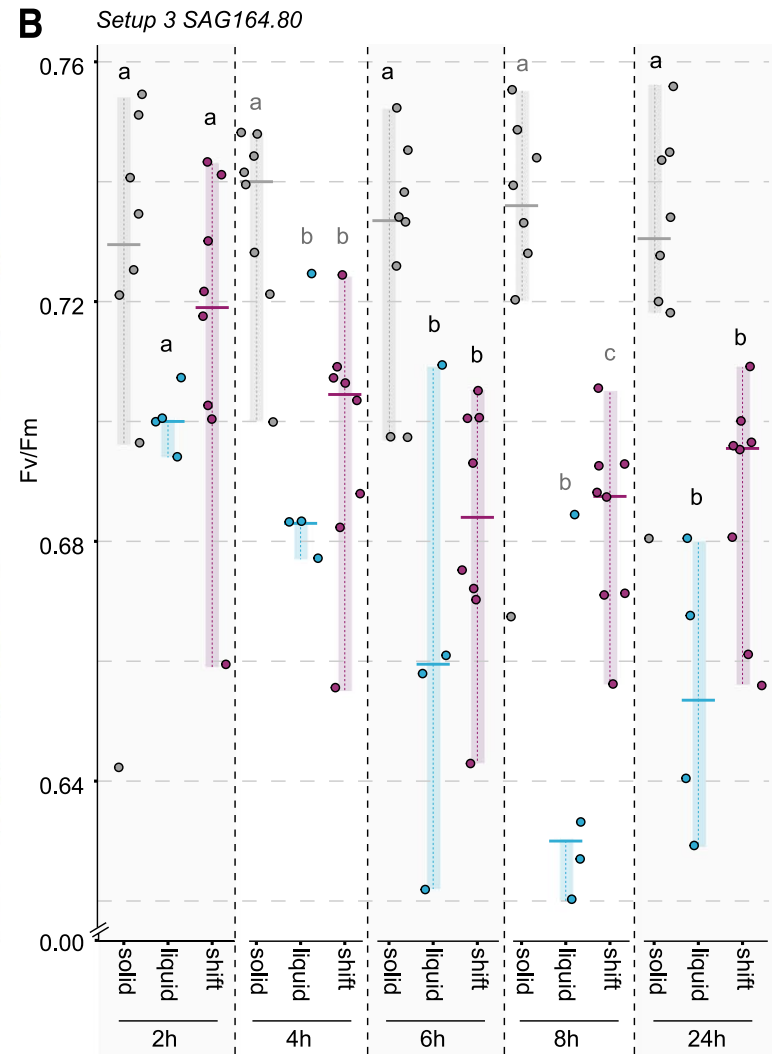

D Setup $4 \mathrm{MZCH} 240$

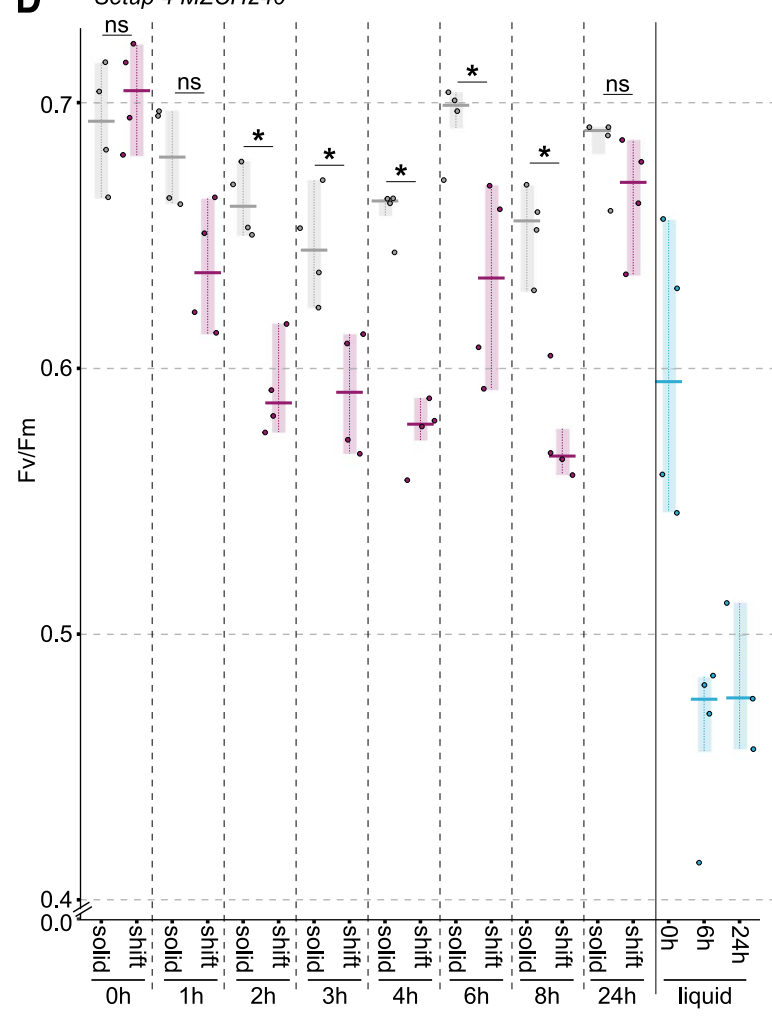

We used BLASTKOALA (Kanehisa et al. 2016) to identify KEGG orthologs among our de novo assembled transcripts and then linked the expression values (fold change) to the corresponding KEGG numbers. All gene expression values for a given KEGG ortholog were summed up as described in de Vries et al. (2020). A KEGG ortholog was considered 
४Fig. 2 Plastid morphology and photophysiological characteristics $\left(F_{\mathrm{v}} / F_{\mathrm{m}}\right)$ in Mougeotia scalaris SAG 164.80 and Mougeotia sp. MZCH 240. A Light micrograph of $M$. scalaris SAG 164.80 in liquid medium. B Maximum PSII quantum yield $\left(F_{\mathrm{v}} / F_{\mathrm{m}}\right)$ in $M$. scalaris SAG 164.80 solid- and liquid-medium control samples (grown for 7 days on WHM-Medium at $20^{\circ} \mathrm{C}, 120 \mu \mathrm{mol}$ quanta $\mathrm{m}^{-2} \mathrm{~s}^{-1}$ ) as well as samples treated with the liquid shift-which were grown on solid medium and submerged in $10 \mathrm{ml}$ liquid medium. C Light micrograph of Mougeotia sp. MZCH $24024 \mathrm{~h}$ after submergence. D $F_{\mathrm{v}} / F_{\mathrm{m}}$ values for Mougeotia sp. MZCH 240 when grown on F/2 medium for 7 days at $22^{\circ} \mathrm{C}, 120 \mu \mathrm{mol}$ quanta $\mathrm{m}^{-2} \mathrm{~s}^{-1}$ on solid and liquid medium. Liquid shift was achieved by adding $10 \mathrm{ml}$ liquid medium to algal cultures grown on solid medium. $F_{\mathrm{v}} / F_{\mathrm{m}}$ values were collected at 0 , $1,2,3,4,6,8$, and $24 \mathrm{~h}$ after the shift and for the control on solid medium. Owing to the low growth rate in liquid medium values for $F_{\mathrm{v}} / F_{\mathrm{m}}$ were measured only at 0,6 , and $24 \mathrm{~h}$ for liquid cultures of Mougeotia sp. MZCH 240. $F_{\mathrm{v}} / F_{\mathrm{m}}$ for $\mathbf{B}$ and $\mathbf{D}$ was measured from the same sample at several time points (from $2 \mathrm{~h}$ up to $24 \mathrm{~h}$ ) after liquid medium was added by using an ImagMAX/L PAM with an IMAG-K5 CCD camera (for details, see the "Material and methods" section). Solid control samples are depicted in grey, liquid control samples are shown in blue, and liquid-treated samples (shift) are depicted in pink. Statistical analysis was done using Mann-Whitney $U$ tests with $\mathrm{R}$ (version 3.6.1); significant differences at $p<0.05$ are depicted using letters and asterisks

up- or downregulated if it had $\mathrm{a} \geq 2$-fold change in gene expression level. $118 \mathrm{KEGG}$ pathways were identified (Figure 1B). In total, expression values for $1677 \mathrm{KEGG}$ orthologs (corresponding 1176 unique KEGG orthologs) were mapped across pathways, among which 90 orthologs were up-regulated and 257 downregulated in Mougeotia sp. MZCH 240 after the shift to liquid conditions; this adds up to a total of 347 responsive KEGG orthologs while 1330 orthologs showed an unchanged response (see the overview in the top right section of Figure 1B).

Most prominent among the top 20 most responsive KEGG pathways were those associated with core metabolic processes such as "oxidative phosphorylation [PATH:ko00190]", "ribosome [PATH:ko03010]", and "amino sugar and nucleotide sugar metabolism [PATH:ko00520]" with 13, 11, and 9 differentially regulated KEGG orthologs respectively. We interpret categories such as ribosome, nucleotide metabolism, and any amino acid metabolism as a readout often observed upon any treatment/shift in environmental conditions: the basal molecular machineries of the cells are responding: they power up for making a range of new/different proteins, resulting in a need to produce a different set of amino acids for making these; prior, as well as alongside of this, they make, process, and transport RNA. Similarly, the downregulation of respiration (oxidative phosphorylation and the citrate cycle) can likely be traced to an overall impacted metabolism. We hence searched whether the data speak to any such process upstream and honed in on photosynthesis - the source of carbon for any photoautotroph.
Two photosynthesis-related pathways, namely, "Porphyrin and chlorophyll metabolism [PATH:ko00860]" (4 ${ }^{\text {th }}$ most responsive, when considering both up- and downregulated KEGG orthologs) and "Photosynthesis [PATH:ko00195]" (16 ${ }^{\text {th }}$ most responsive), contained some of the most highly differentially regulated KEGG orthologs among all 118 pathways; with 4 up- and 5 downregulated KEGG orthologs for the Porphyrin and chlorophyll metabolism pathway and 3 up- and 3 downregulated KEGG orthologs for the photosynthesis pathway (Figure 1C). The finding of photosynthesis-associated genes might explain why other pathways of core metabolism, as well as housekeeping genes are also affected-photosynthesis is at the heart of plant and algal physiology. If the primary fixation of carbon mediated by photosynthesis is affected by a changing environment, it is conceivable that other pathways dependent on the fixed carbon tag along.

\section{Submergence in liquid medium impacts the photophysiology of two strains of Mougeotia}

The top three up- and downregulated KEGG orthologs that belong to the pathway "photosynthesis" mainly fall into the category of photosystem I and II subunits, which suggests pronounced readjustment of the composition and stoichiometry of main components that form the chain of proteins acting in the photosynthetic light reaction; this likely goes hand in hand with selectively elevated turnover rates. We thus honed in on the plastid-associated biology of Mougeotia. For this, we made use of the emerging model system $M$. scalaris SAG 164.80 (Regensdorff et al. 2018; Figure 2A) and investigated its photophysiological changes after submergence using PAM. For this, we used three experimental setups, each with a minimum of three replicates. In a first experimental setup, we tested changes in maximum quantum yield $\left(F_{\mathrm{v}} / F_{\mathrm{m}}\right)$ over time when $M$. $s c a$ laris. was grown on plates and in liquid culture. We initially explored whether photophysiological changes occur over a short period (4h, Figure S1A; setup 1) during daily growth; in the second setup, we investigated whether there are differences in daily performance (24h, Figure S1B; setup 2). On solid medium, $F_{\mathrm{v}} / F_{\mathrm{m}}$ appeared stable when measurements were only $4 \mathrm{~h}$ apart, yet when tested daily, we found a decrease in $F_{\mathrm{v}} / F_{\mathrm{m}}$ in the algal culture ( $p$ value $=0.029$; Figure S1A and B). In liquid culture, $F_{\mathrm{v}} / F_{\mathrm{m}}$ increased from $0.382 \pm 0.020$ to $0.412 \pm 0.018$ after $4 \mathrm{~h}(p$ value $=0.041)$ in setup 1 but was similar to the starting value after $24 \mathrm{~h}$ in setup $2(0.613 \pm 0.017$ to $0.632 \pm 0.015, p$ value $=0.0343$; Figure $\mathrm{S} 1 \mathrm{~A}$ and $\mathrm{B})$. We, however, noted that $F_{\mathrm{v}} / F_{\mathrm{m}}$ of $M$. scalaris SAG 164.80 differed significantly at the first measurement (solid 0h: $p$ value $=0.029$; liquid $0 \mathrm{~h}: p$ value $=0.0095)$. 
Despite differences in the actual values of $F_{\mathrm{v}} / F_{\mathrm{m}}$ in the algal culture, we observed a similar trend after submergence of the algae on plate. Short after submergence $(1 \mathrm{~h}), F_{\mathrm{v}} / F_{\mathrm{m}}$ was similar to that of algal culture grown on non-submerged plates. That said, over time, we saw a decrease of $F_{\mathrm{v}} / F_{\mathrm{m}}$ that significantly differs from that of algae grown on agar after $4 \mathrm{~h}$ (Figure S1 A and B). It is noteworthy, however, that the values between liquid culture, solid culture and the submerged culture are similar at 24h (Figure S1B). The data thus remained inconclusive because only two time points were sampled for liquid- and solid-grown algae and the time points were taken from different cultures.

In a next step, we (i) traced the photophysiological properties of the same liquid-grown, solid-grown and submerged algal cultures over time and (ii) compared the differences in $F_{\mathrm{v}} / F_{\mathrm{m}}$ between the different growth conditions (setup 3; Figure 2B) at a given time point. Both solid and liquid grown cultures remained steady over time in their $F_{\mathrm{v}} / F_{\mathrm{m}}$ (Table 1). In contrast, the submerged cultures tend to have a significantly decreased $F_{\mathrm{v}} / F_{\mathrm{m}}$ after 6,8 , and $24 \mathrm{~h}$ compared to the $F_{\mathrm{v}} / F_{\mathrm{m}}$ at $2 \mathrm{~h}$. This agrees with the decreasing trend observed for $F_{\mathrm{v}} / F_{\mathrm{m}}$ in the first two experiments, where different cultures were measured at the different time points. Additionally, this shows that while the decrease in $F_{\mathrm{v}} / F_{\mathrm{m}}$ for the submergence was real, the differences between the different time points for cultures grown in liquid or on solid medium stems from fluctuations in cultures and culturing.

We next compared the data from a given time point between the different growth conditions. While $F_{\mathrm{v}} / F_{\mathrm{m}}$ did not differ at $2 \mathrm{~h}$, it was always higher in solid grown medium than in liquid and submerged cultures from $4 \mathrm{~h}$ onwards (Figure 2B). Liquid and submerged cultures showed mainly

Table 1 Statistical analysis of maximum quantum yield in M. scalaris SAG 164.80 over time. Numbers denote $p$ values obtained through Mann-Whitney $U$ tests

\begin{tabular}{lllll}
\hline & Solid $2 h$ & Solid $4 h$ & Solid $6 h$ & Solid $8 h$ \\
\hline Solid $4 h$ & 0.1508 & & & \\
Solid $6 h$ & 0.4406 & 0.7789 & & \\
Solid $8 h$ & 0.1484 & 0.726 & 0.7344 & \\
Solid $24 h$ & 0.3828 & 0.1953 & 0.5469 & 0.8332 \\
& Liquid $2 h$ & Liquid $4 h$ & Liquid $6 h$ & Liquid $8 h$ \\
Liquid $4 h$ & 0.875 & & & \\
Liquid $6 h$ & 0.25 & 0.125 & & \\
Liquid $8 h$ & 0.09751 & 0.125 & 0.125 & \\
Liquid $24 h$ & 0.125 & 0.125 & 0.625 & 0.25 \\
& Shift $2 h$ & Shift $4 h$ & Shift $6 h$ & Shift $8 h$ \\
Shift $4 h$ & 0.05469 & & & \\
Shift $6 h$ & $\mathbf{0 . 0 1 5 6 3}$ & 0.07813 & & \\
Shift $8 h$ & $\mathbf{0 . 0 2 9 1 7}$ & 0.07593 & 0.833 & \\
Shift $24 h$ & $\mathbf{0 . 0 2 0 7 1}$ & $\mathbf{0 . 0 3 9 0 6}$ & 0.3615 & 0.5541 \\
\hline
\end{tabular}

similar $F_{\mathrm{v}} / F_{\mathrm{m}}$ values, the only exception being $8 \mathrm{~h}$ after treatment; at this time point, the liquid cultures had a significantly lower $F_{\mathrm{v}} / F_{\mathrm{m}}$ than the submerged culture. Taken together, our data suggest that submerged cultures behaveafter an initial equilibration phase-more similar to cultures grown in liquid medium than on solid medium. While the trend is largely reproducible, cultural fluctuations in initial photosystem performance nevertheless exist.

In order to scrutinize whether the observations we made on Mougeotia scalaris SAG 164.80 (Figure 2A and B) also hold for the strain on which the transcriptomic analyses were performed, we carried out the PAM-based investigations with Mougeotia sp. MZCH 240. The cultures of MZCH 240 had $F_{\mathrm{v}} / F_{\mathrm{m}}$ values at the start of the experiment that were (a)similar for the cultures (grown on solid $1 \%$ agar medium) that were about to be submerged (shift) and those that were kept as the untreated control (solid) $(0.703 \pm 0.017$ (shift) and $0.691 \pm 0.020$ (solid), no significant difference) and (b) comparable to the values of the strain SAG164.80. Cultures of MZCH 240 grown in liquid medium generally had lower $F_{\mathrm{v}} / F_{\mathrm{m}}$ values $[0.598 \pm 0.053$ $\left(t_{0}\right), 0.462 \pm 0.033(6 \mathrm{~h})$, and $\left.0.482 \pm 0.028(24 \mathrm{~h})\right]$. Already after $2 \mathrm{~h}$, submerged cultures had significantly $(p=0.029)$ lower $F_{\mathrm{v}} / F_{\mathrm{m}}$ values; this trend of significantly lower $(p<0.05) F_{\mathrm{v}} / F_{\mathrm{m}}$ values continued at time points $3 \mathrm{~h}, 4 \mathrm{~h}$, $6 \mathrm{~h}$, and $8 \mathrm{~h}$. After $24 \mathrm{~h}$, the submerged cultures appeared to have acclimated to their new culturing conditions as the $F_{\mathrm{v}} / F_{\mathrm{m}}$ values were almost back to $t_{0}: 0.665 \pm 0.019$ (shift) and $0.682 \pm 0.013$ (solid)—with no significant difference. This is in contrast to the physiological behavior of SAG164.80, which did not acclimate to submergence within a $24 \mathrm{~h}$ timeframe. Regardless, it should be re-iterated that MZCH 240 showed significantly lower $F_{\mathrm{v}} / F_{\mathrm{m}}$ values at $4 \mathrm{~h}$ after submergence, which is the time point that was used for transcriptome analyses of this strain; both $\mathrm{MZCH}$ 240 and SAG164.80 behaved alike at this time point with regard to their photophysiology assessed through $F_{\mathrm{v}} / F_{\mathrm{m}}$.

While the photophysiology had recovered at $24 \mathrm{~h}$ after submergence, only then did morphological differences between the solid control and submerged cultures emerge in Mougeotia sp. MZCH 240. The shifted cultures more readily accumulated storage granules (Figure 3); whether these might speak to lipid droplets, as potentially occurring in Spirogyra (see also de Vries and Ischebeck 2020), is unclear. Such granules were sometimes also found in samples of the solid control group. However, the most notable phenotypes were visible in the liquidgrown cultures. Here, we observed rhizoid formation as well as brownish inclusions. Indeed, such inclusions also appeared in solid-grown SAG164.80 as well as liquidgrown SAG 650-1-the latter of which is a strain relative of MZCH 240. Despite them being strain relatives, we noticed that the strain MZCH 240 appeared to have a 


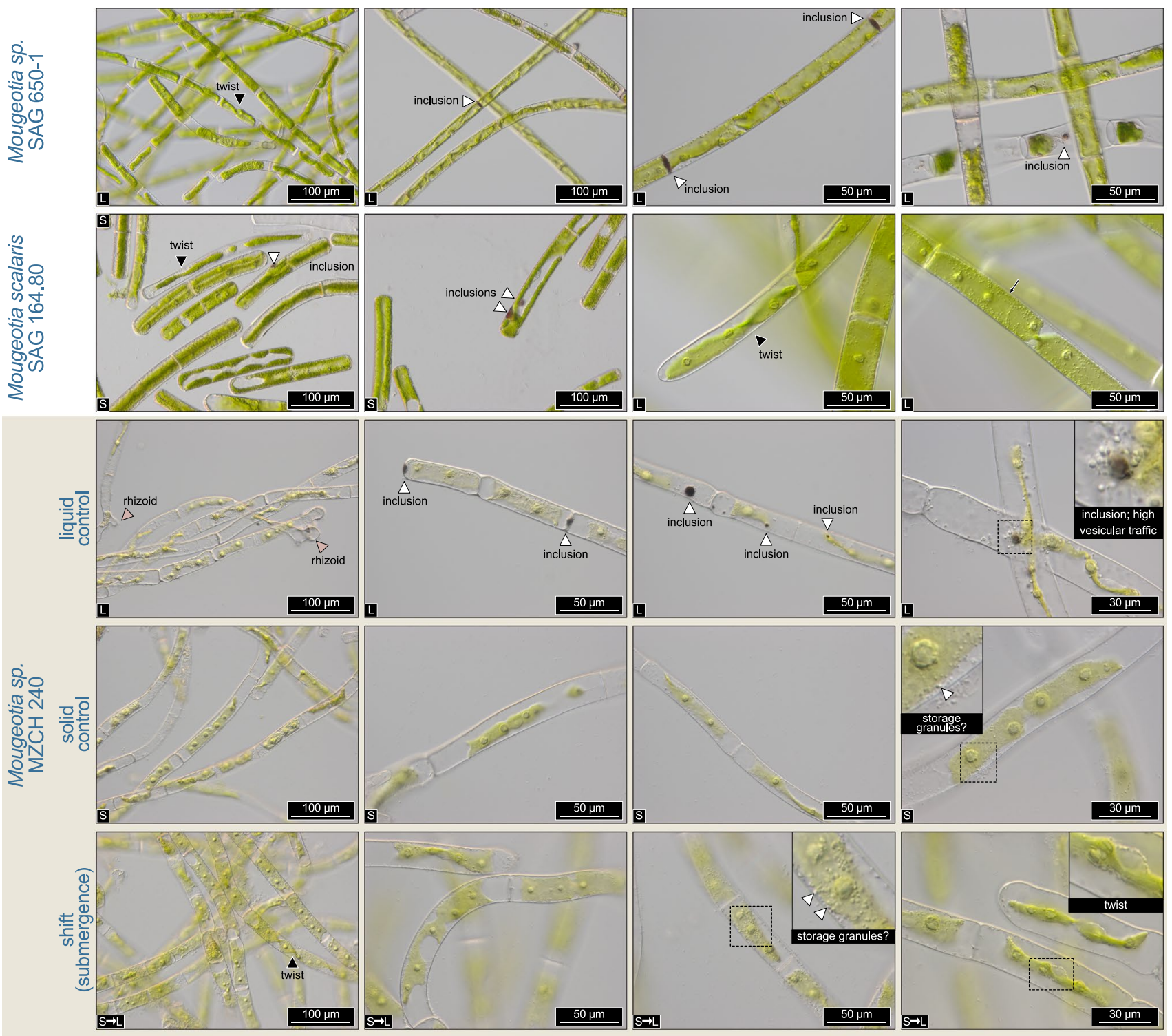

Fig. 3 Notable observations in three Mougeotia strains. Nomarski interference contrast micrographs of the strains Mougeotia sp. SAG 650-1, Mougeotia scalaris SAG 164.80, and Mougeotia sp. MZCH 240; the latter was grown in liquid medium, on solid agar plates, and on agar plates and subjected to $24 \mathrm{~h}$ of submergence in liquid medium ("shift"). The two SAG strains $650-1$ and 164.80 were grown either in liquid or on solid MiEB12 Medium, as indicated by the "L" (liquid medium) or "S" (solid medium) on the bottom left

lighter chlorophyllous hue than SAG 650-1, which is however consistent with our previous experience in culturing MZCH 240 (see de Vries et al. 2020).

Together with the gene expression responses, the photophysiological data highlight the fact that the photosynthetic machinery of Mougeotia responds to the submergence of the algal filaments in liquid medium. We hence next explored which specific genes might be the key players among these changes. side of the pictures. Notable phenotypic observations include: (a) darkly colored inclusions (sometimes co-occurring with high density of intracellular bodies being trafficked); (b) rhizoid formation in liquid culture; (c) formation of granules, possibly for storage. Also note the twisting chloroplasts, including "edge-on" orientations as a sign for functional chloroplast movement induced by microscope illumination. Labels in the bottom left corner denote: L=liquid-grown, $\mathrm{S}=$ solid-grown (agar), $\mathrm{S} \rightarrow \mathrm{L}=$ solid-grown and submerged for $24 \mathrm{~h}$

\section{Responsiveness of genes for light-harvesting components, pigment biosynthesis, and starch metabolism following submergence of Mougeotia sp. MZCH 240}

To understand which gene expression changes were most pronounced upon submergence, we made use of homology searches against the well-annotated genome of Arabidopsis thaliana in combination with the differential transcript 
Fig. 4 Top up-/downregulated genes in Mougeotia sp. MZCH 240 cultured on solid medium and submerged for $4 \mathrm{~h}$ versus control on solid medium. A A heatmap with all up- (red) or downregulated (blue) genes in Mougeotia sp. MZCH 240 based on edgeR analysis of the RNAseq data. Only genes with a significant (Benjamini-Hochberg corrected $p<0.001)$ differential change in gene expression of 2-fold (all differential data are shown as $\log _{2}$ [fold change submergence/control], calculated using edgeR) were considered. Using the $\mathrm{R}$ package pheatmap the data were sorted and $\log _{2}$ values of clusters of genes with the highest/lowest differential gene expression values are shown. The names and descriptions of corresponding Arabidopsis thaliana gene orthologs [prediction based on the reciprocal best BLAST hit (RBBH)] are displayed as well as the corresponding TPM (Transcript per million) values which are shown in a different color gradient (green to yellow). TPM values $>20$ are colored in purple; shift $=$ submergence, ctrl. $=$ control. B Word clouds of the top 50 up(red and orange colors) and top 50 downregulated (blue colors) genes in Mougeotia sp. MZCH 240 generated with Wordle and based on $\log _{2}$ (fold change submergence/control), calculated with edgeR. The words represent the names and/or description of Arabidopsis orthologs (prediction based on the RBBH) and the word size corresponds to the differential gene expression change
A

\begin{tabular}{|l|l|llll||}
\hline & & & \\
\hline & & & & \\
\hline
\end{tabular}

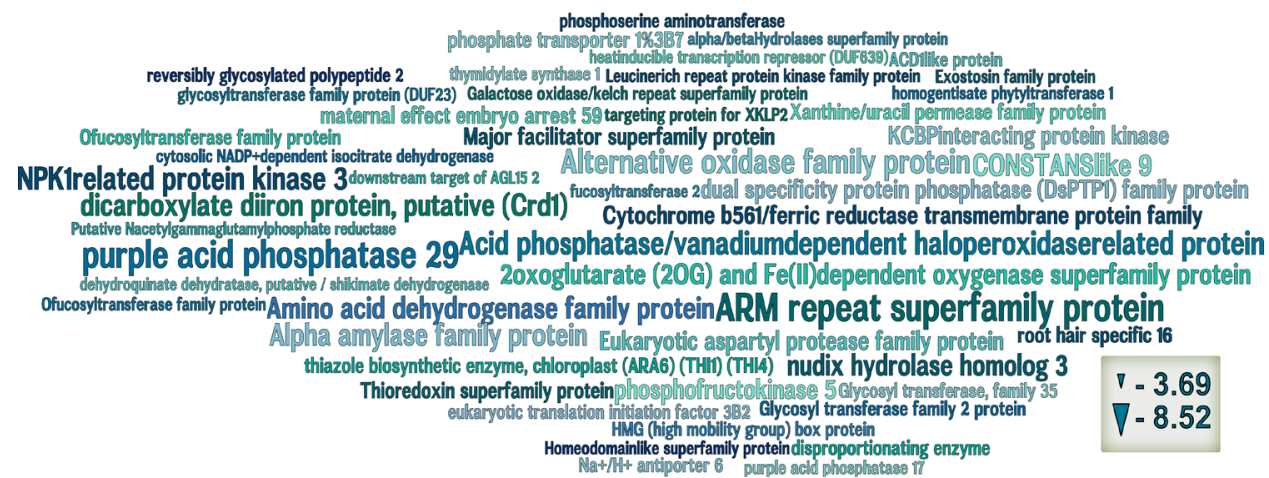

AT5G02290: Protein kinase superfamily protein

AT1 3360 : S-adenosyl-L-methionine-dependent methyltransferases superfamily protein AT3 6020 : acetyl-CoA carboxylase 1

15 AT3G60270: Cupredoxin superfamily protein

AT1G32500: non-intrinsic ABC protein 6

AT5G26130: CAP superfamily protein

A 10

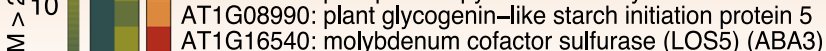

AT3G14840: Leucine-rich repeat transmembrane protein kinase

5 AT1G70860: Polyketide cyclase/dehydrase and lipid transport superfamily protein

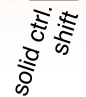

$\mathbf{B}$

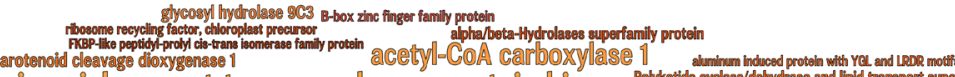

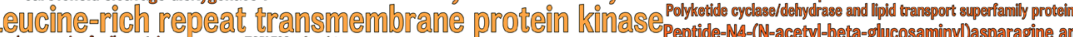

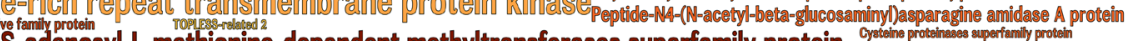

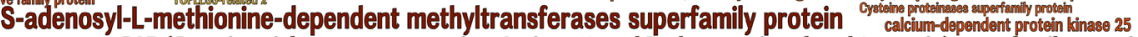

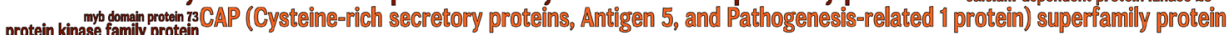
Protein kinase superfamily protein Polyketide cyclase/dehydrase and lipid transport superfamily protein

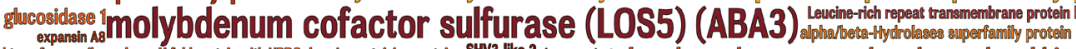

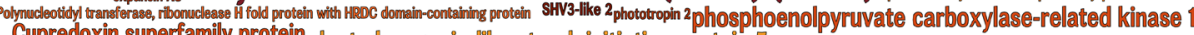

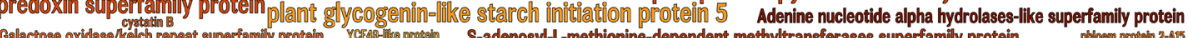

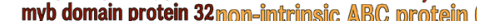

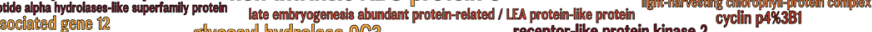

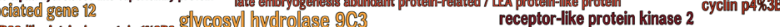

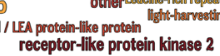

abundance elicited by submergence of Mougeotia sp. MZCH 240. For differential gene expression analyses, we considered only genes that had a Benjamini-Hochberg corrected $p<0.001$ and a differential gene expression change of at least 2-fold (Figure 4). Overall, using these criteria, submergence triggered the upregulation of 120 genes (Table 2) 
Table 2120 transcripts that significantly increased in abundance upon submergence in Mougeotia sp. MZCH 240

\begin{tabular}{|c|c|c|c|c|}
\hline Mousp ID & Best A.t. hit & Annotation & $\log _{2}(\mathrm{FC})$ & FDR \\
\hline Mousp14158_c0_g1_i8 & AT1G16540 & molybdenum cofactor sulfurase (LOS5) (ABA3) & 7.27520966 & $8.0067 \mathrm{E}-24$ \\
\hline Mousp17078_c0_g3_i2 & AT3G14840 & Leucine-rich repeat transmembrane protein kinase & 6.50770827 & $3.1038 \mathrm{E}-22$ \\
\hline Mousp17366_c0_g1_i1 & AT1G70860 & polyketide cyclase/dehydrase and lipid transport & 5.97865925 & $1.8507 \mathrm{E}-17$ \\
\hline Mousp12113_c0_g1_i4 & AT5G02290 & protein kinase superfamily protein & 5.60368112 & $9.185 \mathrm{E}-29$ \\
\hline Mousp17366_c0_g3_i1 & AT1G23360 & S-adenosyl-L-methionine-dependent methyltransferases & 5.40238661 & $9.7774 \mathrm{E}-09$ \\
\hline Mousp17745_c1_g1_i11 & AT1G36160 & acetyl-CoA carboxylase 1 & 5.37604779 & $4.3006 \mathrm{E}-25$ \\
\hline Mousp17215_c0_g5_i2 & AT1G32500 & non-intrinsic $\mathrm{ABC}$ protein 6 & 4.89640786 & $3.5159 \mathrm{E}-10$ \\
\hline Mousp13170_c0_g1_i1 & AT1G12580 & phosphoenolpyruvate carboxylase-related kinase 1 & 4.84629602 & $8.928 \mathrm{E}-17$ \\
\hline Mousp17366_c0_g2_i3 & AT1G08990 & plant glycogenin-like starch initiation protein 5 & 4.80921473 & $1.6156 \mathrm{E}-11$ \\
\hline Mousp15175_c0_g2_i6 & AT5G26130 & Cysteine-rich secretory, Antigen 5, and Pathogenesis-related 1 & 4.76084995 & $9.7774 \mathrm{E}-09$ \\
\hline Mousp14442_c0_g1_i1 & AT3G60270 & Cupredoxin superfamily protein & 4.52531814 & $1.3694 \mathrm{E}-09$ \\
\hline Mousp15384_c0_g2_i2 & AT4G34990 & myb domain protein 32 & 4.09225758 & 4.8567E-33 \\
\hline Mousp17772_c0_g1_i14 & AT4G11050 & glycosyl hydrolase 9C3 & 3.99810327 & $2.8402 \mathrm{E}-10$ \\
\hline Mousp16800_c0_g1_i6 & AT1G67490 & glucosidase 1 & 3.81940181 & $1.062 \mathrm{E}-13$ \\
\hline Mousp17501_c0_g1_i5 & AT3G14920 & Peptide-N4-( $N$-acetyl-beta-glucosaminyl) asparagine amidase A & 3.7028685 & $1.4233 \mathrm{E}-09$ \\
\hline Mousp17241_c0_g1_i6 & AT1G16650 & S-adenosyl-L-methionine-dependent methyltransferases & 3.54376221 & $2.6424 \mathrm{E}-07$ \\
\hline Mousp16885_c0_g1_i12 & AT3G58450 & Adenine nucleotide alpha hydrolases-like & 3.43211036 & $3.7452 \mathrm{E}-12$ \\
\hline Mousp14398_c0_g1_i5 & AT3G63520 & carotenoid cleavage dioxygenase 1 & 3.40138953 & 0.00008287 \\
\hline Mousp17219_c4_g1_i2 & AT5G26150 & protein kinase family protein & 3.27932949 & $2.8364 \mathrm{E}-10$ \\
\hline Mousp12560_c0_g1_i2 & AT2G35890 & calcium-dependent protein kinase 25 & 3.2585986 & $1.3769 \mathrm{E}-06$ \\
\hline Mousp13723_c0_g1_i1 & AT5G45890 & senescence-associated gene 12 & 3.24606174 & $2.5541 \mathrm{E}-09$ \\
\hline Mousp17422_c0_g1_i1 & AT4G11050 & glycosyl hydrolase 9C3 & 3.22808696 & 0.000010803 \\
\hline Mousp17708_c0_g2_i11 & AT3G02130 & receptor-like protein kinase 2 & 3.18914671 & 0.000023637 \\
\hline Mousp15137_c0_g1_i4 & AT2G42450 & alpha/beta-Hydrolases superfamily protein & 3.10782404 & $4.3225 \mathrm{E}-13$ \\
\hline Mousp13988_c0_g1_i5 & none & none & 3.10330699 & $6.974 \mathrm{E}-15$ \\
\hline Mousp16061_c0_g3_i2 & AT1G16250 & Galactose oxidase/kelch repeat & 3.06024435 & $1.1042 \mathrm{E}-16$ \\
\hline Mousp16214_c0_g1_i1 & AT2G44740 & cyclin & 3.05199502 & $2.5095 \mathrm{E}-15$ \\
\hline Mousp14673_c1_g1_i14 & AT1G66970 & SHV3-like 2 & 2.98725544 & $2.3537 \mathrm{E}-07$ \\
\hline Mousp17516_c0_g3_i2 & AT4G20140 & Leucine-rich repeat transmembrane protein kinase & 2.93958975 & 2.9611E-09 \\
\hline Mousp17666_c0_g1_i1 & AT5G20520 & alpha/beta-Hydrolases superfamily protein & 2.89768053 & $1.0882 \mathrm{E}-06$ \\
\hline Mousp16146_c1_g1_i34 & AT1G55960 & Polyketide cyclase/dehydrase and lipid transport & 2.89145041 & $5.3538 \mathrm{E}-08$ \\
\hline Mousp15641_c0_g6_i2 & AT2G32415 & Polynucleotidyl transferase, ribonuclease $\mathrm{H}$ with HRDC domain & 2.78988514 & $9.3814 \mathrm{E}-07$ \\
\hline Mousp15363_c0_g1_i15 & AT5G58140 & phototropin 2 & 2.75782905 & $2.2375 \mathrm{E}-07$ \\
\hline Mousp16839_c0_g1_i4 & AT1G19660 & Wound-responsive family protein & 2.75208096 & 0.000021332 \\
\hline Mousp16811_c0_g3_i2 & AT2G40610 & expansin A8 & 2.73396604 & 0.00082134 \\
\hline Mousp13223_c0_g1_i6 & AT4G18910 & NOD26-like intrinsic protein 1\%3B2 & 2.7251728 & $1.2886 \mathrm{E}-10$ \\
\hline Mousp15049_c1_g5_i1 & AT2G21320 & B-box zinc finger family protein & 2.68212139 & $7.8998 \mathrm{E}-14$ \\
\hline Mousp15748_c2_g2_i3 & AT3G19430 & late embryogenesis abundant & 2.66385694 & $3.9849 \mathrm{E}-08$ \\
\hline Mousp16895_c0_g1_i9 & AT3G19400 & Cysteine proteinases superfamily protein & 2.52775348 & 0.00051691 \\
\hline Mousp17457_c0_g2_i6 & AT3G63190 & ribosome recycling factor, chloroplast precursor & 2.52623134 & $3.4766 \mathrm{E}-12$ \\
\hline Mousp16770_c0_g2_i2 & AT2G34430 & light-harvesting chlorophyll-protein complex II subunit B1 & 2.52608987 & 0.00040624 \\
\hline Mousp17814_c0_g1_i1 & AT3G12490 & cystatin B & 2.51128417 & 3.9795E-09 \\
\hline Mousp15345_c0_g4_i4 & AT3G22850 & aluminum induced protein with YGL and LRDR motifs & 2.49160672 & $8.5789 \mathrm{E}-07$ \\
\hline Mousp16831_c0_g1_i5 & AT4G08850 & Leucine-rich repeat receptor-like protein kinase & 2.47066699 & $2.3962 \mathrm{E}-07$ \\
\hline Mousp12430_c0_g1_i4 & AT4G37260 & myb domain protein 73 & 2.44626078 & $2.1144 \mathrm{E}-07$ \\
\hline Mousp17048_c2_g5_i2 & AT4G22830 & YCF49-like protein & 2.43736515 & $1.4633 \mathrm{E}-09$ \\
\hline Mousp13966_c0_g1_i1 & AT3G16830 & TOPLESS-related 2 & 2.42899009 & 2.509E-07 \\
\hline Mousp12564_c0_g1_i1 & AT3G53000 & phloem protein $2-\mathrm{A} 15$ & 2.4230952 & 3.8864E-10 \\
\hline Mousp15097_c0_g1_i4 & AT2G43560 & FKBP-like peptidyl-prolyl cis-trans isomerase & 2.42256699 & $1.2101 \mathrm{E}-10$ \\
\hline Mousp15383_c0_g1_i2 & AT1G09740 & Adenine nucleotide alpha hydrolases-like & 2.39874357 & 0.00028434 \\
\hline
\end{tabular}


Table 2 (continued)

\begin{tabular}{|c|c|c|c|c|}
\hline Mousp ID & Best A.t. hit & Annotation & $\log _{2}(\mathrm{FC})$ & FDR \\
\hline Mousp17870_c0_g2_i4 & AT2G46580 & Pyridoxamine 5'-phosphate oxidase & 2.39561141 & $1.7197 \mathrm{E}-12$ \\
\hline Mousp15748_c2_g3_i4 & AT3G19430 & late embryogenesis abundant & 2.39052414 & $5.2306 \mathrm{E}-08$ \\
\hline Mousp17536_c0_g2_i15 & AT4G16760 & acyl-CoA oxidase 1 & 2.37665903 & 0.000010184 \\
\hline Mousp17563_c0_g2_i4 & AT1G13980 & sec7 domain-containing protein & 2.36154393 & 0.00012739 \\
\hline Mousp17005_c2_g1_i2 & AT5G54370 & late embryogenesis abundant & 2.34756478 & 0.000050599 \\
\hline Mousp17009_c0_g2_i11 & AT4G33010 & glycine decarboxylase P-protein 1 & 2.34716303 & 0.000027572 \\
\hline Mousp13949_c0_g1_i1 & AT5G19360 & calcium-dependent protein kinase 34 & 2.3443775 & 0.00023592 \\
\hline Mousp14435_c0_g1_i1 & AT3G22750 & Protein kinase superfamily protein & 2.29061994 & $1.2152 \mathrm{E}-11$ \\
\hline Mousp16006_c1_g2_i6 & AT2G15010 & Plant thionin & 2.28071231 & 0.000001202 \\
\hline Mousp17583_c1_g1_i5 & AT1G08550 & non-photochemical quenching 1 & 2.26244193 & $6.0714 \mathrm{E}-06$ \\
\hline Mousp17901_c2_g2_i8 & AT5G14580 & polyribonucleotide nucleotidyltransferase & 2.24801086 & 0.00008991 \\
\hline Mousp15753_c0_g1_i21 & AT2G34260 & transducin family protein / WD-40 repeat & 2.24643147 & $7.4559 \mathrm{E}-07$ \\
\hline Mousp15748_c2_g4_i7 & AT3G19430 & late embryogenesis abundant & 2.24637151 & $6.5626 \mathrm{E}-06$ \\
\hline Mousp15175_c0_g1_i6 & AT2G14610 & pathogenesis-related protein 1 & 2.24594398 & 0.000030745 \\
\hline Mousp17901_c2_g3_i1 & none & none & 2.24550218 & 0.000023275 \\
\hline Mousp16876_c0_g5_i2 & AT3G52140 & tetratricopeptide repeat (TPR)-containing protein & 2.22759061 & 0.000030632 \\
\hline Mousp17754_c1_g2_i1 & AT5G41460 & transferring glycosyl group transferase (DUF604) & 2.22550195 & $1.2101 \mathrm{E}-10$ \\
\hline Mousp10496_c0_g1_i1 & AT4G33880 & ROOT HAIR DEFECTIVE 6-LIKE 2 & 2.21640294 & $7.7321 \mathrm{E}-10$ \\
\hline Mousp14422_c0_g1_i6 & AT1G14870 & PLANT CADMIUM RESISTANCE 2 & 2.21344381 & $8.4799 \mathrm{E}-06$ \\
\hline Mousp13841_c0_g1_i3 & AT2G24440 & selenium binding protein & 2.1868762 & $1.7433 \mathrm{E}-10$ \\
\hline Mousp17685_c0_g1_i2 & AT4G00260 & Transcriptional factor B3 family protein & 2.173197 & 0.00032902 \\
\hline Mousp17103_c0_g2_i3 & AT2G37560 & origin recognition complex second largest subunit 2 & 2.15944614 & 0.000088228 \\
\hline Mousp14784_c2_g1_i6 & AT2G21940 & shikimate kinase 1 & 2.14189653 & $4.7278 \mathrm{E}-09$ \\
\hline Mousp16295_c0_g1_i5 & AT1G31420 & Leucine-rich repeat protein kinase & 2.14136017 & 0.00020268 \\
\hline Mousp17228_c0_g3_i14 & AT2G25185 & Defensin-like (DEFL) family protein & 2.13194267 & 0.00075013 \\
\hline Mousp14776_c0_g1_i2 & AT5G15330 & SPX domain-containing protein 4 & 2.12256734 & $3.8238 \mathrm{E}-10$ \\
\hline Mousp15459_c0_g2_i1 & AT1G44575 & Chlorophyll A-B binding family protein & 2.11945161 & $1.1268 \mathrm{E}-07$ \\
\hline Mousp17556_c0_g1_i6 & AT5G64290 & dicarboxylate transport 2.1 & 2.11755201 & 0.00092571 \\
\hline Mousp16715_c1_g1_i6 & AT2G33855 & transmembrane protein & 2.06354721 & 7.3282E-09 \\
\hline Mousp12292_c0_g1_i2 & AT5G09650 & pyrophosphorylase 6 & 2.02084136 & $1.0549 \mathrm{E}-06$ \\
\hline Mousp15459_c0_g3_i1 & AT1G44575 & Chlorophyll A-B binding family protein & 2.01519992 & $2.6424 \mathrm{E}-07$ \\
\hline Mousp11032_c0_g1_i1 & AT2G36930 & zinc finger $(\mathrm{C} 2 \mathrm{H} 2$ type $)$ family protein & 1.99550424 & $3.5079 \mathrm{E}-07$ \\
\hline Mousp15459_c1_g1_i1 & AT1G44575 & Chlorophyll A-B binding family protein & 1.96857574 & $1.5821 \mathrm{E}-06$ \\
\hline Mousp11772_c0_g1_i3 & AT1G22170 & Phosphoglycerate mutase family protein & 1.96630859 & $1.2869 \mathrm{E}-06$ \\
\hline Mousp17393_c0_g3_i1 & AT2G40490 & Uroporphyrinogen decarboxylase & 1.94764795 & 0.000013015 \\
\hline Mousp15227_c0_g1_i2 & AT5G65230 & myb domain protein 53 & 1.94157129 & $1.3559 \mathrm{E}-06$ \\
\hline Mousp16477_c0_g4_i7 & AT5G52975 & egg cell-secreted-like protein (DUF1278) & 1.92832108 & 0.00010985 \\
\hline Mousp15882_c0_g1_i1 & AT2G19540 & Transducin family protein / WD-40 repeat family protein & 1.92449346 & 0.00096677 \\
\hline Mousp16664_c0_g3_i1 & AT3G12410 & Polynucleotidyl transferase, ribonuclease $\mathrm{H}$-like & 1.91165287 & 0.00028833 \\
\hline Mousp16466_c0_g1_i3 & AT2G35120 & Single hybrid motif superfamily protein & 1.82700212 & $1.5271 \mathrm{E}-06$ \\
\hline Mousp15769_c0_g1_i1 & AT4G24230 & acyl-CoA-binding domain 3 & 1.8206261 & 0.00013362 \\
\hline Mousp16717_c0_g1_i10 & AT3G19430 & late embryogenesis abundant & 1.82053718 & 0.00022751 \\
\hline Mousp17443_c0_g1_i9 & AT4G35000 & ascorbate peroxidase 3 & 1.81353441 & $2.5071 \mathrm{E}-07$ \\
\hline Mousp12426_c0_g1_i4 & AT5G22140 & FAD/NAD(P)-binding oxidoreductase family protein & 1.79593008 & 0.00026667 \\
\hline Mousp15265_c0_g1_i8 & AT5G02160 & transmembrane protein & 1.73674051 & 0.00003623 \\
\hline Mousp14642_c0_g1_i3 & AT4G15520 & tRNA/rRNA methyltransferase (SpoU) family protein & 1.73401135 & 0.00087276 \\
\hline Mousp12053_c0_g1_i1 & AT5G49300 & GATA transcription factor 16 & 1.70744995 & 0.000012895 \\
\hline Mousp17024_c0_g1_i31 & AT1G29900 & carbamoyl phosphate synthetase B & 1.69430823 & 0.00029494 \\
\hline Mousp14546_c0_g1_i2 & AT5G48300 & ADP glucose pyrophosphorylase 1 & 1.67256288 & 0.000055648 \\
\hline Mousp16932_c4_g2_i3 & AT1G78430 & ROP interactive partner 2 & 1.67009459 & 0.00026667 \\
\hline
\end{tabular}


Table 2 (continued)

\begin{tabular}{|c|c|c|c|c|}
\hline Mousp ID & Best A.t. hit & Annotation & $\log _{2}(\mathrm{FC})$ & FDR \\
\hline Mousp17693_c0_g2_i9 & AT5G04270 & DHHC-type zinc finger family protein & 1.6547086 & 0.000030487 \\
\hline Mousp15496_c2_g7_i4 & AT1G20140 & SKP1-like 4 & 1.63691483 & 0.00012795 \\
\hline Mousp14376_c0_g1_i13 & AT3G21150 & B-box 32 & 1.62615568 & 0.00022751 \\
\hline Mousp16045_c0_g1_i2 & AT5G13680 & IKI3 family protein & 1.61640969 & 0.000031256 \\
\hline Mousp17530_c2_g2_i38 & AT3G63380 & ATPase E1-E2 / haloacid dehalogenase-like hydrolase & 1.61216412 & 0.00086341 \\
\hline Mousp13515_c0_g1_i2 & AT5G12180 & calcium-dependent protein kinase 17 & 1.60553028 & 0.000012596 \\
\hline Mousp17689_c0_g1_i7 & AT4G30990 & ARM repeat superfamily protein & 1.59876228 & 0.00092316 \\
\hline Mousp10275_c0_g1_i1 & AT4G14890 & $2 \mathrm{Fe}-2 \mathrm{~S}$ ferredoxin-like superfamily protein & 1.58967395 & 0.0003602 \\
\hline Mousp17110_c0_g2_i14 & AT3G05060 & NOP56-like pre RNA processing ribonucleoprotein & 1.51185847 & 0.00051743 \\
\hline Mousp16289_c1_g3_i6 & AT3G45190 & SIT4 phosphatase-associated family protein & 1.51073535 & 0.00052452 \\
\hline Mousp13265_c0_g1_i2 & AT1G13580 & LAG1 longevity assurance-like protein & 1.47973166 & 0.000049111 \\
\hline Mousp17103_c0_g1_i2 & AT5G48630 & Cyclin family protein & 1.45228723 & 0.00043854 \\
\hline Mousp15997_c0_g1_i6 & AT5G37850 & pfkB-like carbohydrate kinase family protein & 1.45112666 & 0.00029494 \\
\hline Mousp15166_c1_g3_i2 & AT4G27600 & pfkB-like carbohydrate kinase family protein & 1.44542141 & 0.00037996 \\
\hline Mousp17238_c0_g3_i3 & AT3G43520 & Transmembrane proteins $14 \mathrm{C}$ & 1.36066059 & 0.00067169 \\
\hline Mousp16518_c0_g3_i2 & AT4G23890 & $\mathrm{NAD}(\mathrm{P}) \mathrm{H}$-quinone oxidoreductase subunit $\mathrm{S}$ & 1.35071287 & 0.00040989 \\
\hline Mousp17358_c1_g1_i9 & AT3G04460 & peroxin-12 & 1.3027287 & 0.00081849 \\
\hline Mousp17756_c0_g1_i13 & AT4G22890 & PGR5-LIKE A & 1.30095184 & 0.0007896 \\
\hline Mousp13036_c1_g1_i1 & AT4G29350 & profilin 2 & 1.2830376 & 0.00092482 \\
\hline Mousp17102_c1_g1_i1 & AT3G61070 & peroxin $11 \mathrm{E}$ & 1.26175474 & 0.00093898 \\
\hline
\end{tabular}

Differential changes in transcript abundance ( $F C$, fold change) in the samples taken $4 \mathrm{~h}$ after submergence in liquid medium were calculated versus solid control and $\log _{2}$-transformed using edgeR; $F D R$ false discovery rate denotes Benjamini-Hochberg-corrected $p$ values; A.t. Arabidopsis thaliana

and the downregulation of 171 genes (Supplementary Figure S2). Again, photosynthesis-related gene expression patterns stood out - both concerning genes relevant to the light reaction and those of downstream processes, such as three genes putatively coding for chlorophyll a/b-binding proteins (4.3-fold, 4.0-fold, and 3.9-fold upregulation) and a gene putatively encoding a light-harvesting component showed induction (Figure S1; 5.8-fold upregulation).

A Mougeotia sp. transcript homologous to AtABA3 corresponded to the highest gene expression change (i.e. differential change in transcript abundance); it was up-regulated 154.9-fold following the shift from dry to wet. $A B A 3$ codes for a cytosolic molybdenum cofactor sulfurase that converts the carotenoid-derived abscisic aldehyde into the phytohormone abscisic acid (ABA). Despite the fact that several Zygnematophyceae have genes for the ABA receptors (de Vries et al. 2018; Cheng et al. 2019), these likely act in an ABA-independent function (Sun et al. 2019). We interpret the induction of the $A B A 3$ homolog rather as a readout of the aforementioned regulation of pigments (in this case, carotenoid metabolism) and photosynthesis-associated genes expression patterns that impact overall plastid physiology. In line with this, we also found regulation of violaxanthin deepoxidase (4.8-fold upregulation) and a carotenoid cleavage dioxygenase (a homolog of CCD1; 10.6-fold upregulation).
Carotenoid cleavage-derived metabolites are well known signaling molecules in plant cells-especially elicited upon environmental cues (Hou et al. 2016). Indeed, heatinduced changes in the expression of CCDs were observed for Mougeotia sp. (de Vries et al. 2020). Another aspect that needs to be taken into consideration is the adjustment of pigment profiles upon acclimating to a changing habitat; in an aquatic environment, not only the intensity but also the quality of light differs. Here, Mougeotia is a system rich in experimental history: in this algal genus, extensive work on chloroplast movement dependent on light qualities sensed by photoreceptors were carried out (Wagner and Klein 1981). Interestingly, Zygnematophyceae such as Mougeotia stand out by having chimeric photoreceptors containing domains of the red light phytochromes and blue light phototropins, the so-called neochromes (in our assembly Mousp17450 c0_g1; Data S1; (Suetsuga et al. 2006; Li et al. 2015). Responses regulated by these photoreceptors include chloroplast movement (note some of the twisting chloroplasts in Figure 3). We did not find clear signs for the differential regulation of genes related to light quality signaling (e.g. non-significant 2-fold downregulation of the phytochrome B homolog Mousp17540_c0_g1); further, the neochrome transcript Mousp17450_c0_gl was induced upon submergence, with an average TPM of 0.15 in solid control and 0.55 upon 


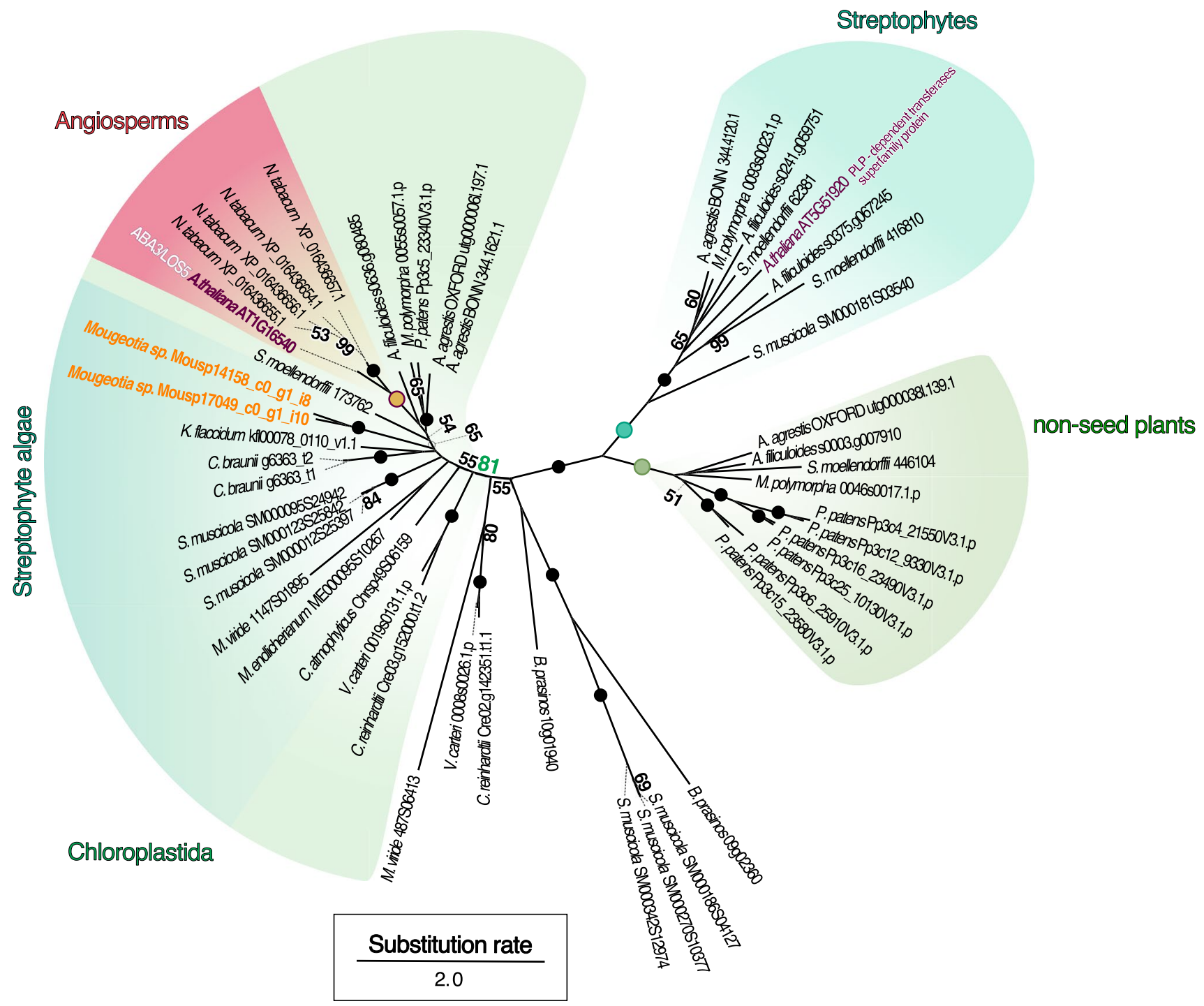

Fig. 5 Phylogenetic framework for the putative ABA3 sequences identified in Mougeotia sp. MZCH 240. Phylogeny of homologs for the molybdenum cofactor sulfurase ABA3. Two homologs of ABA3 (Mousp14158_c0_g1_i8, Mousp17049_c0_g1_i10), the first of which was the most up-regulated gene in Mougeotia sp. MZCH 240 upon submergence, were aligned with 48 ABA3 homologs

4h submergence- - however, as the numbers give away, it was expressed at such a low level that it was excluded from the analyses (see Material and Methods). Overall, it is conceivable that sensing the different spectral qualities of light when shifting to submergence is important and deserves further investigation.

To explore whether the Mougeotia sp. ABA3 homolog we detected is likely an $A B A 3$ ortholog, we performed a phylogenetic analysis. We used BLASTp to mine a phylodiverse protein dataset for ABA3 homologs, MAFFT (Katoh and Standley 2013) to align all putative ABA3 sequences, and IQ-TREE (v1.5.5; Nguyen et al. 2015) to detected in diverse land plants, streptophyte algae, and chlorophyte algae. Homologs were aligned and an unrooted maximum-likelihood phylogeny was computed using $\mathrm{WAG}+\mathrm{F}+\mathrm{I}+\mathrm{G} 4$ (chosen according to BIC) as model for protein evolution and 100 bootstrap replicates. Bootstrap values $<50$ are not shown in the figure; maximum bootstrap support is indicated by a filled dot

construct a maximum likelihood phylogeny (Figure 5). The putative ABA3 homolog detected in Mougeotia sp. (Mousp14158_c0_g1_i8) fell, together with a potential paralog (Mousp17049_c0_g1_i10), into a moderately supported ( $65 \%$ bootstrap value) clade of land plant sequences. This clade was, however, nested in a more highly supported ( $81 \%$ bootstrap) clade of putative molybdenum cofactor sulfurases from across Chloroplastida. Thus, the ABA3 homolog detected in Mougeotia sp. seems to fall into the orthogroup of ABA3-type Molybdenum cofactor sulfurases that is conserved across Chloroplastida. 
Green algae and land plants store photosynthate as starch. The buildup of starch appears to depend on the action of PLANT GLYCOGENIN-LIKE STARCH INITIATION PROTEINs (PGSIP; Chatterjee et al. 2005). Interestingly, we found a homolog of PGSIP5 (AT1G08990) that is strongly induced (28.0-fold up) upon submergence. In light of the changes to the photosynthesis machinery, it is logical to also find genes associated with the downstream buildup of water-insoluble starch; the buildup of reserves appears a common theme among filamentous Zygnematophyceae that are challenged with environmental fluctuations (Pichrtová et al. 2016; Arc et al. 2020; de Vries and Ischebeck 2020). Indeed, the only enriched GO-term process was among the downregulated genes; there, we found that the GO-term "cellular carbohydrate catabolic process" (GO:0044275; $p$ value $6.71 \times 10-4$ ) was enriched.

A homolog of a gene encoding a purple acid phosphatase (PAP) was found as the second most downregulated Mougeotia sp. gene (Mousp11308_c0_g1_i1; 301.0-fold downregulated); the resulting Mougeotia sp. protein bears a signal peptide (likelihood of 0.99 on TargetP-2.0), thus resembling the repertoire of secreted land plant PAPs with diverse functions in response to shifts in environmental conditions and nutrient availability (Bozzo et al. 2002; Kaida et al. 2010; Wang et al. 2011). It is noteworthy that, in a phylogenetic analysis, the Mougeotia sp. PAP fell into a clade of chlorophyte and streptophyte green algae, which formed a monophylum distinct from land plant PAPs (Figure S3).

Finally, we found differentially expressed Mougeotia sp. genes that are classically associated with pathogen response, including a gene putatively encoding a leucine-rich repeat transmembrane protein, (homologs of AT3G14840 and AT4G20140 were 91.0 and 7.7-fold up, respectively) and CAP (Cysteine-rich secretory proteins, Antigen 5, and Pathogenesis-related 1 protein; AT5G26130; 27.1-fold up). Such proteins are, however, equally often a sign of stress elicited by various changes in the environment (Creff et al. 2019 (AT4G20140); Le et al. 2014 (AT3G14840), Chien et al. 2015 (AT5G26130)) - they might simply be a readout of the interwoven network that underpins environmental sensing. In line with this, a gene homologous to protein kinase-encoding AT5G02290 showed clear induction (48.6fold upregulation); this kinase might be involved in various signaling processes and speaks to the response of Mougeotia sp. to the changing environment. Indeed, several genes that speak to a general stress response were up-regulated. These included five LATE EMBRYOGENESIS ABUNDANT (LEA) homologs (6.3-fold, 5.2-fold, 5.1-fold, 4.7-fold, and 3.5-fold up-regulated), which are classical factors responsive to various abiotic stressors in other systems (Ingram and Bartels 1996; Hundertmark and Hincha 2008).

\section{Conclusion}

We observed that submergence of Mougeotia triggered a conspicuous set of differentially regulated genes associated with changes in several photosynthesis and primary carbon metabolic pathways, suggesting remodeling of the photosystem apparatuses. This notion is supported by the observation that (a) various other photosynthesis-associated genes changed their expression and (b) slight but significant changes in the photochemical performance measured through the maximum quantum yield $\left(F_{\mathrm{v}} / F_{\mathrm{m}}\right)$ were observed. Additionally, genes that speak to a remodeling of the pigment composition were regulated. It is conceivable that the composition of accessory pigments is being adjusted in response to the altered quality of light triggered by submergence. Altogether, our data suggest that some of the foremost adjustments that these filamentous zygnematophyceaen algae undergo during dry-to-wet transition are related to photophysiological acclimation; an assessment of the degree to which this holds true in the ecophysiological setting of temporary freshwater bodies is bound to be illuminating.

Supplementary Information The online version contains supplementary material available at https://doi.org/10.1007/s00709-021-01730-1.

Acknowledgements We thank Prof. Dr. Christiane Gatz and Dr. Guido Kriete for giving us access to the ImagMAX/L PAM in the Department of Plant Molecular Biology and Physiology. J.M.R.F.-J. is grateful for being supported by the Ph.D. program "Microbiology and Biochemistry" within the framework of the "Göttingen Graduate Center for Neurosciences, Biophysics, and Molecular Biosciences" (GGNB) at the University of Goettingen.

Funding Open Access funding enabled and organized by Projekt DEAL. Work in the lab of JdV is supported by funding from the European Research Council (ERC) under the European Union's Horizon 2020 research and innovation programme (grant agreement no. 852725; ERC Starting Grant "TerreStriAL"). Research in the lab of JMA was supported by a Discovery Grant from the Natural Sciences and Engineering Research Council of Canada (RGPIN-2014-05871). J.d.V. received grants from the German Research Foundation (DFG) within the framework of the Priority Programme "MAdLand - Molecular Adaptation to Land: Plant Evolution to Change" (SPP 2237; VR 132/41), in which J.M.R.F.-J partakes as associate member. K.v.S. received grants from Deutsche Forschungsgemeinschaft (Schw687/13-1).

Data availability All data generated or analyzed during this study are included in this published article (and its supplementary information files), and the public databases of the NCBI: all RNAseq read data have been uploaded to the NCBI SRA. The reads from the control samples are available under the run IDs SRR9083693, SRR9083694, SRR9083695, SRR9083697, SRR9083698, SRR9083699; liquid treatment is available under the run IDs SRR9083681, SRR9083682, SRR9083688 (https://www.ncbi.nlm.nih.gov/sra?term=SRP198800). The reference assembly is publicly available under NCBI BioProject PRJNA543475 (https://www.ncbi.nlm.nih.gov/bioproject/PRJNA 543475). 
Open Access This article is licensed under a Creative Commons Attribution 4.0 International License, which permits use, sharing, adaptation, distribution and reproduction in any medium or format, as long as you give appropriate credit to the original author(s) and the source, provide a link to the Creative Commons licence, and indicate if changes were made. The images or other third party material in this article are included in the article's Creative Commons licence, unless indicated otherwise in a credit line to the material. If material is not included in the article's Creative Commons licence and your intended use is not permitted by statutory regulation or exceeds the permitted use, you will need to obtain permission directly from the copyright holder. To view a copy of this licence, visit http://creativecommons.org/licenses/by/4.0/.

\section{References}

Arc E, Pichrtová M, Kranner I, Holzinger A (2020) Pre-akinete formation in Zygnema sp. from polar habitats is associated with metabolite re-arrangement. J Exp Bot 57:289-289. https://doi. org/10.1093/jxb/eraa123

Banks JA, Nishiyama T, Hasebe M et al (2011) The selaginella genome identifies genetic changes associated with the evolution of vascular plants. Science 332:960-963. https://doi.org/10.1126/scien ce. 1203810

Bolger AM, Lohse M, Usadel B (2014) Trimmomatic: a flexible trimmer for Illumina sequence data. Bioinformatics 30:2114-2120. https://doi.org/10.1093/bioinformatics/btu170

Bowman JL, Kohchi T, Yamato KT et al (2017) Insights into Land Plant Evolution Garnered from the Marchantia polymorpha Genome. Cell 171:287-299.e15. https://doi.org/10.1016/j.cell. 2017.09.030

Bozzo GG, Raghothama KG, Plaxton WC (2002) Purification and characterization of two secreted purple acid phosphatase isozymes from phosphate-starved tomato (Lycopersicon esculentum ) cell cultures: secreted acid phosphatases of $\mathrm{P}_{\mathrm{i}}$-starved tomato cells. Eur J Biochem 269:6278-6286. https://doi.org/10. 1046/j.1432-1033.2002.03347.x

Chatterjee Manash, Berbezy P, Vyas D, Coates S, Barsby T (2005) Reduced expression of a protein homologous to glycogenin leads to reduction of starch content in Arabidopsis leaves. Plant Science 168(2):501-509. https://doi.org/10.1016/j.plant sci.2004.09.015

Cheng S, Xian W, Fu Y et al (2019) Genomes of subaerial Zygnematophyceae provide insights into land plant evolution. Cell 179:1057-1067.e14. https://doi.org/10.1016/j.cell.2019.10.019

Chien PS, Nam HG, Chen YR (2015) A salt-regulated peptide derived from the CAP superfamily protein negatively regulates salt-stress tolerance in Arabidopsis. J Exp Bot 66:5301-5313. https://doi.org/10.1093/jxb/erv263

Christa G, Cruz S, Jahns P et al (2017) Photoprotection in a monophyletic branch of chlorophyte algae is independent of energydependent quenching (qE). New Phytol 214:1132-1144. https:// doi.org/10.1111/nph.14435

Correa-Galvis V, Poschmann G, Melzer M et al (2016) PsbS interactions involved in the activation of energy dissipation in Arabidopsis. Nat Plants 2:15225. https://doi.org/10.1038/nplants. 2015.225

Creff A, Brocard L, Joubès J, Taconnat L, Doll NM, Marsollier AC, Ingram $\mathrm{G}$ et al (2019) A stress-response-related inter-compartmental signalling pathway regulates embryonic cuticle integrity in Arabidopsis. PLoS Genet 15:1-28. https://doi.org/10.1371/ journal.pgen.1007847 de Vries J, Ischebeck T (2020) Ties between stress and lipid droplets pre-date seeds. Trends Plant Sci 25:1203-1214. https://doi.org/ 10.1016/j.tplants.2020.07.017

de Vries PJR, Simons J, van Beem AP (1983) Sporopollenin in the spore wall of Spirogyra (Zygnemataceae, Chlorophyceae). Acta Botanica Neerlandica 32:25-28. https://doi.org/10.1111/j.14388677.1983.tb01674.x

de Vries J, de Vries S, Slamovits CH et al (2017) How embryophytic is the biosynthesis of phenylpropanoids and their derivatives in streptophyte algae? Plant Cell Physiol 58:934-945. https://doi. org/10.1093/pcp/pcx037

de Vries J, Curtis BA, Gould SB, Archibald JM (2018) Embryophyte stress signaling evolved in the algal progenitors of land plants. Proc Natl Acad Sci U S A 115:E3471-E3480. https://doi.org/ 10.1073/pnas. 1719230115

de Vries J, Vries S, Curtis BA et al (2020) Heat stress response in the closest algal relatives of land plants reveals conserved stress signaling circuits. Plant J 324:1064-1024. https://doi.org/10. $1111 /$ tpj. 14782

de Vries S, Fürst-Jansen JMR, Irisarri I, Dhabalia Ashok A, Ischebeck T, Feussner K, Abreu IN, Petersen M, Feussner I, de Vries J (2021) The evolution of the phenylpropanoid pathway entailed pronounced radiations and divergences of enzyme families. Plant J 107:975-1002. https://doi.org/10.1111/tpj.15387

Eden E, Navon R, Steinfeld I et al (2009) GOrilla: a tool for discovery and visualization of enriched GO terms in ranked gene lists. BMC Bioinform 10:48. https://doi.org/10.1186/1471-2105-10-48

FASTQC (2018) A quality control tool for high throughput sequence data. Available at www.bioinformatics.babraham.ac.uk/projects/ fastqc. Accessed September 15, 2018.

Foyer CH, Lelandais M, Kunert KJ (1994) Photooxidative stress in plants. Physiol Plant 92:696-717. https://doi.org/10.1111/j.13993054.1994.tb03042.x

Friedl T, Lorenz M (2012) The Culture Collection of Algae at Göttingen University (SAG): a biological resource for biotechnological and biodiversity research. Procedia Environ Sci 15:110-117. https://doi.org/10.1016/j.proenv.2012.05.015

Fürst-Jansen JMR, de Vries S, de Vries J (2020) Evo-physio: on stress responses and the earliest land plants. J Exp Bot 66:4-16. https:// doi.org/10.1093/jxb/eraa007

Gerotto C, Morosinotto T (2013) Evolution of photoprotection mechanisms upon land colonization: evidence of PSBS-dependent NPQ in late Streptophyte algae. Physiol Plant 149:583-598. https://doi. org/10.1111/ppl.12070

Guillard RRL (1975) Culture of phytoplankton for feeding marine invertebrates. In: Smith WL, Chanley MH (eds) Culture of marine invertebrate animals. Plenum Book Publ. Corp, New York, pp 29-60

Haas BJ, Papanicolaou A, Yassour M et al (2013) De novo transcript sequence reconstruction from RNA-seq using the Trinity platform for reference generation and analysis. Nat Protoc 8:1494-1512. https://doi.org/10.1038/nprot.2013.084

Herburger K, Holzinger A (2015) Localization and Quantification of Callose in the Streptophyte Green Algae Zygnema and Klebsormidium: Correlation with Desiccation Tolerance. Plant Cell Physiol 56:2259-2270. https://doi.org/10.1093/pcp/pcv139

Holzinger A, Pichrtová M (2016) Abiotic stress tolerance of charophyte green algae: new challenges for omics techniques. Front Plant Sci 7:273-217. https://doi.org/10.3389/fpls.2016.00678

Holzinger A, Kaplan F, Blaas K, Zechmann B, Komsic-Buchmann K, Becker B (2014) Transcriptomics of desiccation tolerance in the streptophyte green alga Klebsormidium reveal a land plant-like defense reaction. PLoS One 9:e110630

Holzinger A, Albert A, Aigner S, Uhl J, Schmitt-Kopplin P, Trumhová K, Pichrtová M (2018) Arctic, antarctic, and temperate green algae Zygnema spp. under UV-B stress: vegetative cells perform 
better than pre-akinetes. Protoplasma 255:1239-1252. https://doi. org/10.1007/s00709-018-1225-1

Hori K, Maruyama F, Fujisawa T et al (2014) Klebsormidium flaccidum genome reveals primary factors for plant terrestrial adaptation. Nat Commun 5:3978. https://doi.org/10.1038/ncomms4978

Hou X, Rivers J, León P et al (2016) Synthesis and function of apocarotenoid signals in plants. Trends Plant Sci 21:792-803. https:// doi.org/10.1016/j.tplants.2016.06.001

Hundertmark M, Hincha DK (2008) LEA (Late Embryogenesis Abundant) proteins and their encoding genes in Arabidopsis thaliana. BMC Genomics 9:118-122. https://doi.org/10.1186/ 1471-2164-9-118

Hutin C, Nussaume L, Moise N et al (2003) Early light-induced proteins protect Arabidopsis from photooxidative stress. Proc Natl Acad Sci U S A 100:4921-4926. https://doi.org/10.1073/pnas. 0736939100

Ingram J, Bartels D (1996) The molecular basis of dehydration tolerance in plants. Annu Rev Plant Physiol Plant Mol Biol 47:377403. https://doi.org/10.1146/annurev.arplant.47.1.377

Irisarri I, Darienko T, Pröschold T, Fürst-Jansen JMR, Jamy M, de Vries J (2021) Unexpected cryptic species among streptophyte algae most distant to land plants. Proc R Soc B 288:20212168. https://doi.org/10.1098/rspb.2021.2168

Jahns P, Holzwarth AR (2012) The role of the xanthophyll cycle and of lutein in photoprotection of photosystem II. BBA-Bioenergetics 1817:182-193. https://doi.org/10.1016/j.bbabio.2011.04.012

Jiao C, Sørensen I, Sun X et al (2020) The Penium margaritaceum Genome: Hallmarks of the Origins of Land Plants. Cell 181(P1097-1111):E12. https://doi.org/10.1016/j.cell.2020.04.019

Kaida R, Serada S, Norioka N et al (2010) Potential role for purple acid phosphatase in the dephosphorylation of wall proteins in tobacco cells. Plant Physiol 153:603-610

Kalyaanamoorthy S, Minh BQ, Wong TKF et al (2017) ModelFinder: fast model selection for accurate phylogenetic estimates. Nat Methods 14:587-589. https://doi.org/10.1038/nmeth.4285

Kanehisa M, Sato Y, Morishima K (2016) BlastKOALA and GhostKOALA: KEGG tools for functional characterization of genome and metagenome sequences. J Mol Biol 428:726-731. https://doi. org/10.1016/j.jmb.2015.11.006

Karsten U, Lembcke S, Schumann R (2007) The effects of ultraviolet radiation on photosynthetic performance, growth and sunscreen compounds in aeroterrestrial biofilm algae isolated from building facades. Planta 225:991-1000

Karsten U, Herburger K, Holzinger A (2014) Dehydration, temperature, and light tolerance in members of the aeroterrestrial green algal genus Interfilum (Streptophyta) from biogeographically different temperate soils. J Phycol 50:804-816. https://doi.org/10.1111/ jpy. 12210

Katoh K, Standley DM (2013) MAFFT Multiple Sequence Alignment Software Version 7: improvements in performance and usability. Mol Biol Evol 30:772-780. https://doi.org/10.1093/molbev/ mst010

Kitzing C, Karsten U (2015) Effects of UV radiation on optimum quantum yield and sunscreen contents in members of the genera Interfilum, Klebsormidium, Hormidiella and Entransia (Klebsormidiophyceae, Streptophyta). Eur J Phycol 50:279-287. https:// doi.org/10.1080/09670262.2015.1031190

Krause GH, Vernotte C, Briantais JM (1982) Photoinduced quenching of chlorophyll fluorescence in intact chloroplasts and algae. Resolution into two components. Biochim Biophys Acta 679:116-124. https://doi.org/10.1016/0005-2728(82)90262-6

Lamesch P, Berardini TZ, Li D et al (2012) The Arabidopsis Information Resource (TAIR): Improved gene annotation and new tools. Nucleic Acids Res 40:D1202-D1210. https://doi.org/10.1093/nar/ gkr1090
Lang D, Ullrich KK, Murat F et al (2018) The Physcomitrella patenschromosome-scale assembly reveals moss genome structure and evolution. Plant J 93:515-533. https://doi.org/10.1111/tpj.13801

Le MH, Cao Y, Zhang X-C, Stacey G (2014) LIK1, A CERK1-Interacting Kinase, Regulates Plant Immune Responses in Arabidopsis. PLoS ONE 9(7):e102245. https://doi.org/10.1371/journal.pone. 0102245

Leebens-Mack JH, Barker MS, Carpenter EJ et al (2019) One thousand plant transcriptomes and the phylogenomics of green plants. Nature 574:679-685. https://doi.org/10.1038/s41586-019-1693-2

Li B, Dewey CN (2011) RSEM: accurate transcript quantification from RNA-Seq data with or without a reference genome. BMC Bioinform 12:323. https://doi.org/10.1186/1471-2105-12-323

Li X-P, Björkman O, Shih C, Grossman AR, Rosenquist M, Jansson S, Niyogi KK (2000) A pigment-binding protein essential for regulation of photosynthetic light harvesting. Nature 403:391-395. https://doi.org/10.1038/35000131

Li F-W, Melkonian M, Rothfels CJ et al (2015) Phytochrome diversity in green plants and the origin of canonical plant phytochromes. Nat Commun 6:7852. https://doi.org/10.1038/ncomms8852

Li F-W, Nishiyama T, Waller M et al (2020) Anthoceros genomes illuminate the origin of land plants and the unique biology of hornworts. Nature Plants 6:259-272. https://doi.org/10.1038/ s41477-020-0618-2

Li F-W, Brouwer P, Carretero-Paulet L, Cheng S, de Vries J, Delaux P-M et al (2018) Fern genomes elucidate land plant evolution and cyanobacterial symbioses. Nature Plants 4(7):460-472. https:// doi.org/10.1038/s41477-018-0188-8

Mann HB, Whitney DR (1947) On a test of whether one of two random variables is stochastically larger than the other. Ann Math Stat:50-60

Merchant SS, Prochnik SE, Vallon O et al (2007) The Chlamydomonas genome reveals the evolution of key animal and plant functions. Science 318:245-251. https://doi.org/10.1126/science.1143609

Mikhailyuk T, Glaser K, Holzinger A, Karsten U (2015) Biodiversity of Klebsormidium (Streptophyta) from alpine biological soil crusts (Alps, Tyrol, Austria, and Italy). J Phycol 51:750-767. https://doi. org/10.1111/jpy.12316

Montané MH, Dreyer S, Triantaphylidès C, Kloppstech K (1997) Early light-inducible proteins during long-term acclimation of barley to photooxidative stress caused by light and cold: High level of accumulation by posttranscriptional regulation. Planta 202:293-302. https://doi.org/10.1007/s004250050131

Moreau H, Verhelst B, Couloux A et al (2012) Gene functionalities and genome structure in Bathycoccus prasinos reflect cellular specializations at the base of the green lineage. Genome Biol 13:R74

Morris JL, Puttick MN, Clark JW et al (2018) The timescale of early land plant evolution. Proc Natl Acad Sci U S A 115:E2274E2283. https://doi.org/10.1073/pnas.1719588115

Müller P, Li XP, Niyogi KK (2001) Non-photochemical quenching. A response to excess light energy. Plant Physiol 125:1558-1566. https://doi.org/10.1186/gb-2012-13-8-r74

Nguyen L-T, Schmidt HA, von Haeseler A, Minh BQ (2015) IQ-TREE: a fast and effective stochastic algorithm for estimating maximumlikelihood phylogenies. Mol Biol Evol 32:268-274. https://doi. org $/ 10.1093 / \mathrm{molbev} / \mathrm{msu} 300$

Nichols HW (1973) Growth media - freshwater. In: Stein JR (ed) Handbook of Phycological Methods. Cambridge University Press, London, pp 16-17

Nishiyama T, Sakayama H, de Vries J et al (2018) The Chara Genome: secondary complexity and implications for plant terrestrialization. Cell 174:448-464.e24. https://doi.org/10.1016/j.cell.2018.06.033

Ohama N, Sato H, Shinozaki K, Yamaguchi-Shinozaki K (2017) Transcriptional regulatory network of plant heat stress response. Trends Plant Sci 22:53-65 
Peers G, Truong TB, Ostendorf E et al (2009) An ancient light-harvesting protein is critical for the regulation of algal photosynthesis. Nature 462:518-521. https://doi.org/10.1038/nature08587

Permann C, Herburger K, Niedermeier M, Felhofer M, Gierlinger N, Holzinger A (2021) Cell wall characteristics during sexual reproduction of Mougeotia sp. (Zygnematophyceae) revealed by electron microscopy, glycan microarrays and RAMAN spectroscopy. Protoplasma 258(6):1261-1275. https://doi.org/10.1007/ s00709-021-01659-5

Pichrtová M, Remias D, Lewis LA, Holzinger A (2013) Changes in phenolic compounds and cellular ultrastructure of arctic and antarctic strains of Zygnema (Zygnematophyceae, Streptophyta) after exposure to experimentally enhanced UV to PAR ratio. Microb Ecol 65:68-83

Pichrtová M, Arc E, Stöggl W, Kranner I, Hájek T, Hackl H, Holzinger A (2016) Formation of lipid bodies and changes in fatty acid composition upon pre-akinete formation in Arctic and Antarctic Zygnema(Zygnematophyceae, Streptophyta) strains. FEMS Microbiol Ecol 92:fiw096-fiw099. https://doi.org/10.1093/femsec/fiw096

Poulícková A, Zizka Z, Hasler P, Benada O (2007) Zygnematalean zygospores: morphological features and use in species identification. Folia Microbiol 52:135-145. https://doi.org/10.1007/BF029 32152

Prochnik SE, Umen J, Nedelcu AM et al (2010) Genomic analysis of organismal complexity in the multicellular green alga Volvox carteri. Science 329:223-226. https://doi.org/10.1126/science. 1188800

Regensdorff M, Deckena M, Stein M, Borchers A, Scherer G, Lammers M, Hänsch R, Zachgo S, Buschmann H (2018) Transient genetic transformation of Mougeotia scalaris (Zygnematophyceae) mediated by the endogenous $\alpha$-tubulin1 promoter. J Phycol 54:840-849

Renault H, Werck-Reichhart D, Weng J-K (2019) Harnessing lignin evolution for biotechnological applications. Curr Opin Biotechnol 56:105-111. https://doi.org/10.1016/j.copbio.2018.10.011

Rippin M, Becker B, Holzinger A (2017) Enhanced desiccation tolerance in mature cultures of the streptophytic green alga Zygnema circumcarinatum revealed by transcriptomics. Plant Cell Physiol 58:2067-2084. https://doi.org/10.1093/pcp/pcx136

Rippin M, Pichrtová M, Arc E et al (2019) Metatranscriptomic and metabolite profiling reveals vertical heterogeneity within a Zygnemagreen algal mat from Svalbard (High Arctic). Environ Microbiol 21:4283-4299. https://doi.org/10.1111/1462-2920.14788

Robinson MD, Oshlack A (2010) A scaling normalization method for differential expression analysis of RNA-seq data. Genome Biol 11:R25. https://doi.org/10.1186/gb-2010-11-3-r25

Robinson MD, McCarthy DJ, Smyth GK (2010) edgeR: A Bioconductor package for differential expression analysis of digital gene expression data. Bioinformatics 26:139-140. https://doi.org/10. 1093/bioinformatics/btp616

Schlösser UG (1994) SAG - Sammlung von Algenkulturen at the University of Göttingen Catalogue of Strains 1994. Botanica Acta 107(3):113-186. https://doi.org/10.1111/j.1438-8677.1994.tb007 84.x

Sierro N, Battey JND, Ouadi S et al (2014) The tobacco genome sequence and its comparison with those of tomato and potato. Nat Commun 5:3883. https://doi.org/10.1038/ncomms4833

Suetsuga N, Mittmann F, Wagner G, Hughes J, Wada M (2006) A chimeric photoreceptor gene, NEOCHROME, has arisen twice during plant evolution. Proc Natl Acad Sci U S A 102:13705-13709

Sun Y, Harpazi B, Wijerathna-Yapa A et al (2019) A ligand-independent origin of abscisic acid perception. Proc Natl Acad Sci U S A 116:24892-24899. https://doi.org/10.1073/pnas.1914480116

von Schwartzenberg K, Bornfleth S, Lindner A-C, Hanelt D (2013) The Microalgae and Zygnematophyceae Collection Hamburg (MZCH) - living cultures for research on rare streptophytic algae. Algol Stud 142:77-107

Wagner G, Klein K (1981) Mechanism of chloroplast movement in Mougeotia. Protoplasma 109:169-185. https://doi.org/10.1007/ BF01287638

Wang L, Li Z, Qian W et al (2011) The arabidopsis purple acid phosphatase AtPAP10 is predominantly associated with the root surface and plays an important role in plant tolerance to phosphate limitation. Plant Physiol 157:1283-1299. https://doi.org/10.1104/ pp. 111.183723

Wang S, Li L, Li H et al (2020) Genomes of early-diverging streptophyte algae shed light on plant terrestrialization. Nature Plants 6:95-106. https://doi.org/10.1038/s41477-019-0560-3

Wickett NJ, Mirarab S, Nguyen N et al (2014) Phylotranscriptomic analysis of the origin and early diversification of land plants. Proc Natl Acad Sci U S A 111:E4859-E4868. https://doi.org/10.1073/ pnas. 1323926111

Wodniok S, Brinkmann H, Glöckner G et al (2011) Origin of land plants: do conjugating green algae hold the key? BMC Evol Biol 11:104. https://doi.org/10.1186/1471-2148-11-104

Zimmer A, Lang D, Richardt S et al (2007) Dating the early evolution of plants: detection and molecular clock analyses of orthologs. Mol Gen Genomics 278:393-402. https://doi.org/10.1007/ s00438-007-0257-6

Publisher's note Springer Nature remains neutral with regard to jurisdictional claims in published maps and institutional affiliations. 